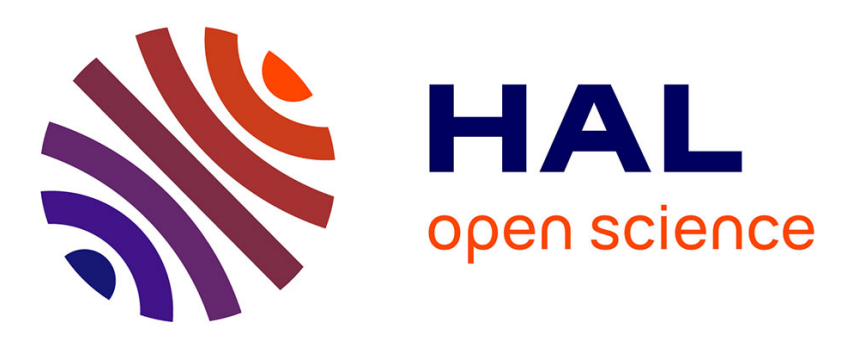

\title{
Evolution of species trait through resource competition
}

Sepideh Mirrahimi, Benoît Perthame, Joe Yuichiro Wakano

\section{To cite this version:}

Sepideh Mirrahimi, Benoît Perthame, Joe Yuichiro Wakano. Evolution of species trait through resource competition. Journal of Mathematical Biology, 2012, 64 (7), pp.1189-1223. 10.1007/s00285011-0447-z . hal-00566888

\section{HAL Id: hal-00566888 \\ https://hal.science/hal-00566888}

Submitted on 17 Feb 2011

HAL is a multi-disciplinary open access archive for the deposit and dissemination of scientific research documents, whether they are published or not. The documents may come from teaching and research institutions in France or abroad, or from public or private research centers.
L'archive ouverte pluridisciplinaire HAL, est destinée au dépôt et à la diffusion de documents scientifiques de niveau recherche, publiés ou non, émanant des établissements d'enseignement et de recherche français ou étrangers, des laboratoires publics ou privés. 


\title{
Evolution of species trait through resource competition
}

\author{
Sepideh Mirrahimi * Benoît Perthame ${ }^{* \dagger} \quad$ Joe Yuichiro Wakano ${ }^{\ddagger}$ \\ February 17, 2011
}

\begin{abstract}
To understand the evolution of diverse species, theoretical studies using a Lotka-Volterra type direct competition model had shown that concentrated distributions of species in continuous trait space often occurs. However, a more mechanistic approach is preferred because the competitive interaction of species usually occurs not directly but through competition for resource. We consider a chemostat-type model where species consume resource that are constantly supplied. Continuous traits in both consumer species and resource are incorporated. Consumers utilize resource whose trait values are similar with their own. We show that, even when resource-supply has a continuous distribution in trait space, a positive continous distribution of consumer trait is impossible. Self-organized generation of distinct species occurs. We also prove global convergence to the evolutionarily stable distribution.
\end{abstract}

Key-Words: Adaptive dynamics; Continuous trait; Ecological competition for resource; Dirac concentrations, asymptotic methods.

\section{Introduction}

Evolutionary origin of species diversity has been one of the most important problems in evolutionary ecology. Individuals with similar traits (e.g. body size or shape) face strong competition, which might lead to the adaptive radiation. As a model of such competition-driven speciation, several theoretical studies have shown that the species distribution in continuous trait space will often evolve toward single peak or multiple peaks that are distinct from each other (see [22, 7, 4, 18, 12]). This provides a mechanism of evolution of diverse but distinct species in nature. These studies strengthen the theory of speciation by Adaptive Dynamics that usually assumes distinct species a priori [10, 9, 16].

Most of the previous results are derived from a simple model that assumes direct species competition of Lotka-Volterra type. On the other hand, competitive interaction among species generally occurs in competition for resource such as prey or nutrient. For example, birds with similar beak shapes are in competition because they utilize similar food resource. Another example is found in ecological stoichiometry where consumer species with similar C:N:P (carbon: nitrogen: phosphorus ratio)

\footnotetext{
*Université P. et M. Curie Paris 06, CNRS UMR 7598, Laboratoire Jacques-Louis Lions, F-75252 Paris cedex 05. Email: mirrahimi@ann.jussieu.fr

${ }^{\dagger}$ INRIA EPI BANG and Institut Universitaire de France. Email: benoit.perthame@upmc.fr

${ }^{\ddagger}$ Meiji Institute for Advanced Study of Mathematical Sciences and PRESTO, Japan Science and Technology Agency. Email: joe@math.meiji.ac.jp
} 
requirements experience competition for nutrient supply with their optimal C:N:P ratio (see [24]). Thus, the competitive interaction should be modeled not directly but implicitly through the resource competition. Here we study a model of evolution of traits based on this approach. Mathematically, this corresponds to assuming a resource-consumption kernel, instead of a direct competition kernel.

From Volterra-Gause's competitive exclusion principle (see [13, 23] and the references therein), it is well established that in a model with $N$ limiting factors (e.g. nutrients) at most $N$ species can generically survive. In the context of continuous traits $N$ species is represented by the sum of at most $N$ Dirac masses. For $N$ small $(1,2, \ldots)$, these states are stable. Is this true asymptotically as $N$ becomes large? A way to answer this question is to study also the case of a continuous distribution of resource according to a parameter related to the predation ability of the population.

In this paper, we first describe our model of species competition through resource dynamics (section 2). Both consumer trait and resource trait take continuous values. First we study the condition for the survival of population (section 3 ) and the forms of steady states (section 4). In section 5, we introduce the notion of Evolutionary Stable Distribution and show nonlinear stability for large times. Our method is based on Lyapunov functionals and in particular our result covers the convergence to polymorphic ESD. To illustrate our results, we show an example case where we choose specific function form. In sections 6 and 7 we give details on the transient to polymorphic states.

\section{Model}

\begin{tabular}{|c|l|}
\hline$x$ & Consumer species trait (evolving) \\
\hline$y$ & Resource trait \\
\hline$n(x, t)$ & Consumer species distribution \\
\hline$r(x)$ & Trait dependent growth rate \\
\hline$R(y, t)$ & Resource distribution \\
\hline$R_{i n}(y)$ & Trait dependent resource-supply rate \\
\hline$m_{1}(x)$ & Mortality of consumer species \\
\hline$m_{2}(y)$ & Decay rate of resource \\
\hline$K(x, y)$ & Consumption rate of resource $y$ by individuals of trait $x$ \\
\hline$\varepsilon$ & Mutation rate \\
\hline
\end{tabular}

Table 1: The notations used for the chemostat models (11) and (2).

Our model is denoted as

$$
\left\{\begin{array}{l}
\partial_{t} n(x, t)=n(x, t)\left(-m_{1}(x)+r(x) \int K(x, y) R(y, t) d y\right)+\varepsilon \Delta n(x, t), \\
\partial_{t} R(y, t)=-m_{2}(y) R(y, t)+R_{i n}(y)-R(y, t) \int r(x) K(x, y) n(x, t) d x
\end{array}\right.
$$

and with initial data

$$
n(0, x)=n^{0}(x) \geq 0, \quad R(0, y)=R^{0}(y) \geq 0,
$$

for $x \in \mathbb{R}$ and $y \in \mathbb{R}$. Here $n(x, t) \geq 0$ is consumer species density with trait $x$ and $R(y, t) \geq 0$ is resource density with trait $y$. Resource is constantly supplied at rate $R_{i n}(y)>0$ which drives 
the system (a chemostat type model). Trait-dependent mortalities (or chemostat outflow rate or resource decay rate) are denoted by $m_{1}(x)$ and $m_{2}(y)$. Consumption of resource is denoted by a trait dependent efficiency $r(x)$ and by a resource-consumption kernel $K(x, y)$ that defines how species with trait $x$ depends on resource with trait $y$. Without loss of generality, we normalize $K(x,$.$) so that r(x)$ represents a net growth rate

$$
K(x, y) \geq 0, \quad \int K(x, y) d y=1 .
$$

We consider the evolution of a consumer trait, $x$. The mutation rate is denoted by $\varepsilon$ in (1). Our system is a generalized MacArthur's model for species packing in niche space (see [15]). It can also be viewed as a model of population dynamics in spatially heterogeneous environment where resource-supply rate, resource decay rate, and consumer mortality are function of spatial position, $x$ (see [21]).

Our system consists of two species; consumer and resource. In general, two species reaction-diffusion equations are difficult to analyze. Nevertheless, our results in sections 3 , 4 and 5 are valid for the full system (1) with unspecified functional forms. To obtain even stronger results in sections 6 and 7 , we will later focus on a reduced system by taking the quasi-static approximation of resource dynamics as follows

$$
\left\{\begin{array}{l}
\partial_{t} n(x, t)=n(x, t)\left(-m_{1}(x)+r(x) \int K(x, y) R(y, t) d y\right)+\varepsilon \Delta n(x, t), \\
R(y, t)=\frac{R_{i n}(y)}{m_{2}(y)+\int r(x) K(x, y) n(x, t) d x} .
\end{array}\right.
$$

The basic idea comes from the assumption that changes in species composition occur slower in consumer population than in resource population. This is because the reproduction and death of consumer species has, in many cases, slower time scale than those of prey species or resource dynamics of nutrient particles.

Finally we introduce a particular case that we will use throughout the paper to illustrate more intuitively the results; this is when $r(x), m_{1}(x)$ and $m_{2}(y)$ are constant and when the resource-supply distribution and the resource-consumption kernel are gaussians:

$$
\begin{gathered}
K(x, y)=\frac{1}{\sigma_{K} \sqrt{2 \pi}} \exp \left(-\frac{|x-y|^{2}}{2 \sigma_{K}^{2}}\right), \quad R_{i n}(y)=\frac{M_{i n}}{\sigma_{i n} \sqrt{2 \pi}} \exp \left(-\frac{y^{2}}{2 \sigma_{i n}^{2}}\right), \\
m_{1}(x) \equiv \bar{m}_{1}, \quad m_{2}(y) \equiv \bar{m}_{2}, \quad \bar{m}_{0}=\min \left(\bar{m}_{1}, \bar{m}_{2}\right) \quad r(x) \equiv r .
\end{gathered}
$$

We assume the data are smooth enough, Lipschitz continuous and we use the following notations and assumptions

$$
\begin{gathered}
\underline{m}_{1}=\inf _{x} m_{1}(x)>0, \quad \underline{m}_{2}=\inf _{x} m_{2}(y)>0, \quad \underline{m}_{0}=\min \left(\underline{m}_{1}, \underline{m}_{2}\right), \quad r_{M}=\sup _{x} r(x), \\
r_{0}=\inf _{x} r(x)>0, \quad r_{2}=\sup _{x}\left|r_{x}(x)\right|, \\
M_{1}=\sup _{x}\left|m_{1, x}(x)\right|, \quad M_{2}=\sup _{x}\left|m_{2, x}(x)\right|, \\
M^{0}=\int n^{0}(x) d x+\int R^{0}(y) d y, \\
R_{1}=\sup _{y} R_{i n}(y), \quad R_{2}=\sup _{y}\left|\nabla R_{i n}(y)\right|, \\
K_{M}=\max _{x, y} K(x, y), \quad M_{i n}=\int R_{i n}(y) d y, \quad R_{i n} \in L^{2}(\mathbb{R}),
\end{gathered}
$$




$$
\begin{gathered}
K_{1}=\int r(x) \int K(x, y) \frac{R_{\text {in }}(y)}{m_{2}(y)} d y d x, \\
K_{2}=\sup _{x, y}\left|K_{x}(x, y)\right|, \quad K_{3}=\sup _{x, y}\left|K_{y}(x, y)\right|, \\
K_{4}=\sup _{x, y}\left|\Delta_{x}(r(x) K(x, y))\right| .
\end{gathered}
$$

We call the population is '(strictly) monomorphic' at $x=\bar{x}$ if $n(x)=0$ for any $x \neq \bar{x}$ and '(strictly) polymorphic' if $n(x)=0$ for any $x$ except for $x=\bar{x}_{1}, \bar{x}_{2}, \ldots$. On the other hand, we call the population is 'approximately monomorphic' if the distribution has single peak with tails. Similarly, 'approximately polymorphic' population consists of multiple peaks in its trait distribution (multimodal), see Figure1.
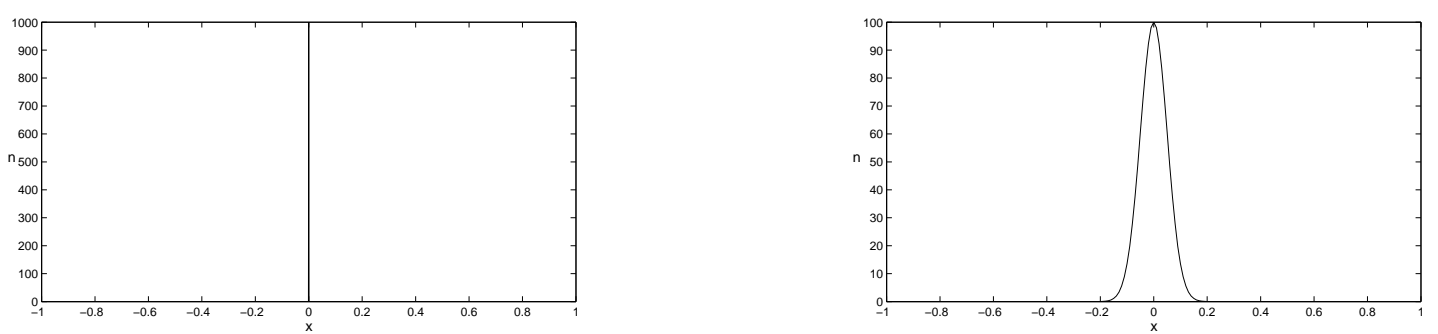

Figure 1: Left: a strictly monomorphic population. Right: an approximately monomorphic population.

\section{Survival condition}

We study whether consumer species can survive on a given rate of resource-supply in models (1) and (2). We begin with two preliminary observations: a balance law for the total flow holds which implies estimates on the total population. Next, we prove the necessary condition for survival, which implies the existence of the threshold level of resource-supply below which species go extinct, that is they reach the consumer-free steady state

$$
\overline{\bar{n}}(x):=0, \quad \overline{\bar{R}}(y):=\frac{R_{\text {in }}(y)}{m_{2}(y)} .
$$

We also prove that the population does not grow up to infinity; there is an upper bound.

\subsection{Fundamental balance laws}

Integrating and summing the two lines in the full system (10), we obtain the following balance law for the total flow

$$
\frac{d}{d t}\left[\int n(x, t) d x+\int R(y, t) d y\right]=\int R_{i n}(y) d y-\left[\int m_{1}(x) n(x, t) d x+\int m_{2}(y) R(y, t) d y\right] .
$$

This indicates that the total biomass is finite and positive. Therefore competition for resource is indeed at work.

In the case of system (2) the biomass balance becomes

$$
\frac{d}{d t} \int n(x, t) d x=\int R_{\text {in }}(y) d y-\left[\int m_{1}(x) n(x, t) d x+\int m_{2}(y) R(y, t) d y\right] .
$$




\subsection{Boundedness of the total population}

These balance laws have the consequence that the population remains bounded.

Theorem 3.1 We define $\rho(t)=\int n(x, t) d x$. Then we have, for both systems (11) and (2)

$$
\rho(t) \leq \max \left(M^{0}, \frac{M_{i n}}{\underline{m}_{0}}\right):=\rho_{M},
$$

with $M^{0}, M_{i n}$ and $\underline{m}_{0}$ defined in (7), (9) and (44). Moreover we have, with the notation (13),

$$
R(y, t) \leq \max (R(y, 0), \overline{\bar{R}}(y)) .
$$

Proof. Proof of (16): For the full system (11), using the total biomass relation (14),

$$
\frac{d}{d t}\left[\int n(x, t) d x+\int R(y, t) d y\right] \leq M_{i n}-\underline{m}_{0}\left[\int n(x, t) d x+\int R(y, t) d y\right] .
$$

Thus we obtain that

$$
\frac{d}{d t}\left(\int n(t, x) d x+\int R(t, y) d y\right) \leq 0, \quad \text { if } \quad \frac{M_{i n}}{\underline{m}_{0}} \leq \int n(t, x) d x+\int R(t, y) d y .
$$

We deduce that $\int n(t, x) d x+\int R(t, y) d y \leq \max \left(M^{0}, \frac{M_{i n}}{\underline{m}_{0}}\right)$. Hence (16)).

In the case of system (2), using (15) we obtain that

$$
\frac{d}{d t} \int n(t, x) d x \leq M_{i n}-\underline{m}_{1} \int n(t, x) d x .
$$

It follows that $\rho(t) \leq \max \left(\int n^{0}(x) d x, \frac{M_{\text {in }}}{\underline{m}_{1}}\right) \leq \max \left(M^{0}, \frac{M_{i n}}{\underline{m}_{0}}\right)$.

Proof of (17): For the full system (1), we have $\frac{d}{d t} R(y, t) \leq 0$ if $R(y, t) \leq \frac{R_{i n}(y)}{m_{2}(y)}$. Thus (17). In the case of the system (2) the proof is immediate.

\subsection{Necessary condition for survival}

We prove that the system gets extinct if the mortality/decay rates $m_{1}$ and $m_{2}$ are too large, see Figure 2. This is the regime

Theorem 3.2 (Condition for survival) We assume (44), (77), the first part of (8), (19) and (12). For the solutions to systems (1) or (2), if $\int \overline{\bar{R}}(y)\left|\ln R^{0}(y)\right| d y<\infty$ and

$$
m_{1}(x) \geq r(x) \int K(x, y) \overline{\bar{R}}(y) d y, \quad \text { for all } x \in \mathbb{R}
$$

then the solution gets extinct, that is $\int n(x, t) d x$ vanishes and $R(y, t)$ converges a.e. to $\overline{\bar{R}}(y)$ in (13) .

Otherwise the system survives, i.e. $\int n(x, t) d x$ does not vanish, at least when there are no mutations and the initial data $n^{0}$ is positive everywhere. 

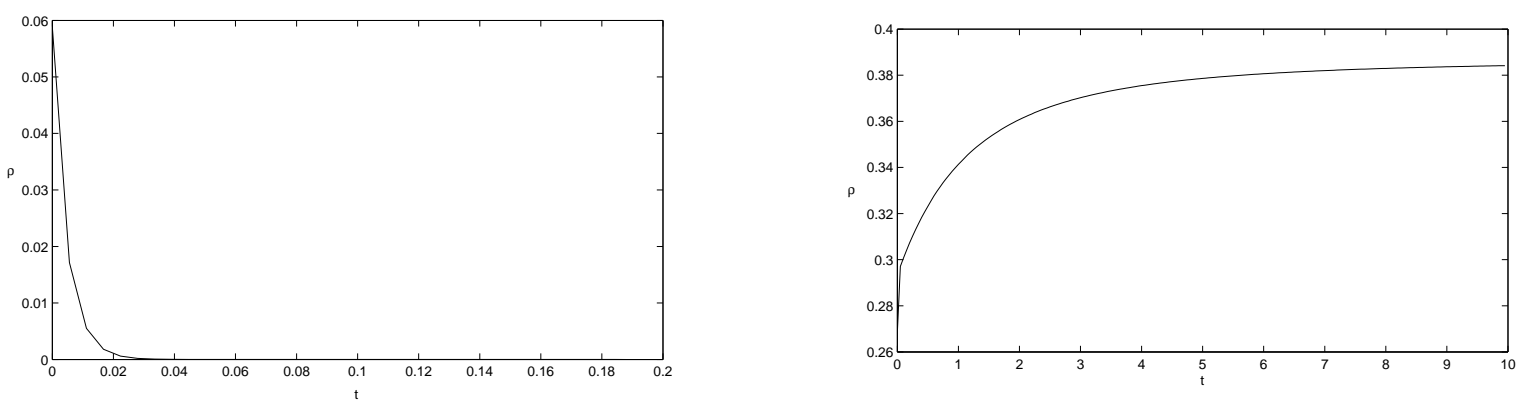

Figure 2: Here the horizontal axis is time and the vertical axis is $\rho$ the total mass. The model studied here is the one given in (2) in the particular case of (3) with $\varepsilon=0.001, \sigma_{K}=0.6, \sigma_{i n}=1, r=1$, $M_{i n}=3$. Left: the mortality rates $\bar{m}_{1}=\bar{m}_{2}=1.1$ are too large such that the non-extinction condition (19) is not respected and we observe extinction in finite time. Right: the mortality terms $\bar{m}_{1}=\bar{m}_{2}=1$ are small enough such that the non-extinction condition (19) is respected and this avoids extinction. This is in accordance with the results in Theorem 3.2.

This condition can be made explicit for the gaussian case (3) and gives

$$
\bar{m}_{1} \bar{m}_{2} \geq \frac{r M_{i n}}{\sqrt{2 \pi\left(\sigma_{K}^{2}+\sigma_{i n}^{2}\right)}} .
$$

Proof. (i) We assume that the assumption (18) is satisfied and prove extinction.

For the system (11): We first prove that as $t$ goes to $+\infty, R(y, t)$ converges to $\overline{\bar{R}}(y)$ using the following Lyapunov functional

$$
\overline{\bar{S}}(t)=-\int \overline{\bar{R}}(y) \ln R(y, t) d y+\int n(x, t) d x+\int R(y, t) d y .
$$

We differentiate $\overline{\bar{S}}$ and obtain

$$
\frac{d \overline{\bar{S}}}{d t}(t)=-\int \frac{m_{2}(y)}{R(y, t)}(\overline{\bar{R}}(y)-R(y, t))^{2} d y-\int n(x, t)\left(m_{1}(x)-r(x) \int K(x, y) \overline{\bar{R}}(y) d y\right) d x
$$

Thanks to assumption (18), we obtain that $\frac{d \overline{\bar{S}}}{d t}$ is always negative and thus $\overline{\bar{S}}$ is a decreasing function with respect to $t$. Using Theorem 3.1 we deduce that $\overline{\bar{S}}(t)$ is bounded from below so that

$$
\int_{0}^{\infty} \int \frac{m_{2}(y)}{R(y, t)}(\overline{\bar{R}}(y)-R(y, t))^{2} d y d t<+\infty .
$$

Because $R$ is bounded from above thanks to Theorem 3.1, we conclude that

$$
Q(t):=\int(\overline{\bar{R}}(y)-R(y, t))^{2} d y \quad \text { satisfies } \quad \int_{0}^{\infty} Q(t) d t<\infty .
$$

But we can estimate, with the notation $K * n(y)=\int r(x) K(x, y) n(x, t) d x$

$$
\begin{aligned}
\frac{1}{2} Q^{\prime}(t) & =-\int(\overline{\bar{R}}(y)-R(y, t)) \frac{\partial R(y)}{\partial t} d y \\
& =-\int\left((\overline{\bar{R}}(y)-R(y, t))\left(m_{2} \overline{\bar{R}}(y)-m_{2} R(y, t)-R K * n(y)\right) d y\right. \\
& =-\int\left((\overline{\bar{R}}(y)-R(y, t))^{2}\left(m_{2}+K * n(y)\right) d y-\int((\overline{\bar{R}}(y)-R(y, t)) \overline{\bar{R}}(y) K * n(y) d y .\right.
\end{aligned}
$$


Because $0 \leq K * n(y) \leq K_{M} \rho_{M}$, we conclude that

$$
\left|Q^{\prime}(t)\right| \leq C \int\left((\overline{\bar{R}}(y)-R(y, t))^{2}+\int \overline{\bar{R}}(y)^{2} d y \in L^{1}+L^{\infty}(0,+\infty)\right.
$$

With the fact that $Q \in L^{1}\left(\mathbb{R}^{+}\right)$, this is enough to conclude that $Q(t) \rightarrow 0$ as $t \rightarrow \infty$ and thus that

$$
\lim _{t \rightarrow \infty} R(y, t)=\overline{\bar{R}}(y), \quad \text { a.e. } \quad \text { and } \quad \text { in } L^{1} \cap L^{2}(\mathbb{R})
$$

Notice that $R(t)$ is 'regularized' since initially it needs not be square integrable.

It remains to prove that $n$ vanishes. We first conclude from the previous step that $\frac{\partial R(y, t)}{\partial t}$ converges to 0 in the distribution sense. Therefore, as $t \rightarrow \infty$,

$$
R(y, t) \int r(x) K(x, y) n(x, t) d x \rightarrow 0 \quad \text { weakly. }
$$

From assumptions (41), (7), (9) and (12) we notice that $\left|\frac{d}{d t} \int r(x) K(x, y) n(x, t) d x\right|$ is bounded. Therefore, using the above convergence, we find that

$$
\int \overline{\bar{R}}(y) \int r(x) K(x, y) n(x, t) d x d y \rightarrow 0 \quad \text { pointwise. }
$$

Because $K$ and $\overline{\bar{R}}(y)$ are positive, this means that $n$ vanishes locally not necessariliy in $L^{1}(\mathbb{R})$. To conclude, we notice that the dissipation term in the right hand side of (20) also vanishes (weakly but also strongly by regularity as before). Therefore we conclude that $\int m_{1}(x) n(x, t) d x$ vanishes and the result is proved.

For the system (21): We integrate the first equation, use (18) and arrive at

$$
\frac{d}{d t} \int n(x, t) d x=\int n(x, t)\left(-m_{1}(x)+r(x) \int K(x, y) R(y, t) d y\right) d x \leq 0 .
$$

Therefore $\int n(x, t) d x$ is decreasing and

$$
0 \leq \int_{0}^{\infty} \int n(x, t)\left(m_{1}(x)-r(x) \int K(x, y) R(y, t) d y\right) d x d t<+\infty .
$$

Using (18) it follows that

$$
0 \leq \int_{0}^{\infty} \int\left(\int r(x) n(x, t) K(x, y) d x\right)\left(\frac{R_{\text {in }}(y)}{m_{2}(y)}-\frac{R_{\text {in }}(y)}{m_{2}(y)+\int r(x) n(x, t) K(x, y) d x}\right) d y d t<+\infty .
$$

Since $\int r(x) n(x, t) K(x, y) d x$ is bounded, convexity implies that

$$
\int_{0}^{\infty} \int\left(\int r(x) n(x, t) K(x, y) d x\right)^{2} R_{i n}(y) d y d t<\infty .
$$

Since $\left|\frac{d}{d t} \int r(x) K(x, y) n(x, t) d x\right|$ is bounded, following the arguments for the case of the system (11) we obtain successively that

$$
\lim _{t \rightarrow \infty} R(y, t)=\overline{\bar{R}}(y) \quad \text { a.e., } \quad \text { and } \quad \int n(x, t) d x \underset{t \rightarrow \infty}{\longrightarrow} 0 .
$$


(ii) Now we assume that the assumption (18) is not satisfied, i.e. for some $\bar{x} \in \mathbb{R}$ we have

$$
m_{1}(\bar{x})<r(\bar{x}) \int K(\bar{x}, y) \frac{R_{i n}(y)}{m_{2}(y)} d y .
$$

We prove that if there is no mutation, i.e. $\varepsilon=0$, the solutions to systems (11) and (2) do not get extinct.

For system (11): We assume that $\int n(x, t) d x \underset{t \rightarrow \infty}{\longrightarrow} 0$. Therefore

$$
\int r(x) n(x, t) K(x, y) d x \underset{t \rightarrow \infty}{\longrightarrow} 0 .
$$

It follows that

$$
\partial_{t} R(y, t)=-m_{2}(y) R(y, t)+R_{\text {in }}(y)+\beta(t)
$$

with $\beta(t) \underset{t \rightarrow \infty}{\longrightarrow} 0$. We deduce that

$$
R(y, t) \underset{t \rightarrow \infty}{\longrightarrow} \overline{\bar{R}}(y) .
$$

Using the dominated convergence Theorem and the boundedness of $K(\cdot, \cdot)$ we obtain that $\int K(x, y) R(y, t) d y$ converges to $\int K(x, y) \overline{\bar{R}}(y) d y$, locally uniformly in $x$ as $t \rightarrow+\infty$. Therefore for $x$ in a neighborhood of $\bar{x}$ for $t>t_{0}$ with $t_{0}$ large enough, using system (11) with $\varepsilon=0$ and using (22), we have

$$
\begin{aligned}
\partial_{t} n(x, t) & =n(x, t)\left(-m_{1}(x)+r(x) \int K(x, y) R(y, t) d y\right) \\
& \approx n(x, t)\left(-m_{1}(x)+r(x) \int K(x, y) \overline{\bar{R}}(y) d y\right) \\
& >\text { an }(x, t),
\end{aligned}
$$

with $a$ a positive constant. We deduce that $n(x, t)$ blows up when $t \rightarrow \infty$ in a neighborhood of $\bar{x}$. This is in contradiction with $\int n(x, t) d x \rightarrow 0$.

For system (22): In this case we deduce directly from (23) that $R(y, t) \underset{t \rightarrow \infty}{\longrightarrow} \overline{\bar{R}}(y)$, using the definition of $R(y, t)$. The other steps are as above.

\section{$4 \quad$ Steady states}

Next question we address concerns the possible shapes of stationary solutions for the model (1) but neglecting the mutation term. Firstly, in section 4.1 we show that under some general assumptions, there is no positive continuous steady solution. This distinguishes this model from that of LotkaVolterra with direct competition where positive steady states can generally occur depending on the model coefficients (see [8, 4, 12, 22]). Secondly, in Section 4.2 we show that there may exist steady solutions in the form of Dirac masses. This confirms that the model is convenient to observe the high population concentrations as Dirac deltas. It means that one or several specific traits can survive while the other traits disappear.

In this section, we always consider the model without mutations that is system (1) with $\varepsilon=0$. Then a steady state $(\bar{n}, \bar{R})$ satisfies

$$
\int \bar{R}(y) K(x, y) d y=\frac{m_{1}(x)}{r(x)} \quad \forall x \in \operatorname{Supp} \bar{n}, \quad \bar{R}(y)\left(m_{2}(y)+\int r(x) K(x, y) \bar{n}(x) d x\right)=R_{\text {in }}(y) .
$$




\subsection{Positive steady states}

We say that the population is at positive steady state, if consumer species density $\bar{n}(x)$ is positive for all trait values.

To analyse possible solutions, we use assumptions (10), (4) and the second part of (9). In the one hand we have, from the second equality in (24) and these assumptions

$$
r(x) \int K(x, y) \bar{R}(y) d y d x \leq r(x) \int K(x, y) \frac{R_{i n}(y)}{m_{2}(y)} d y d x:=K_{1}<+\infty .
$$

In the other hand we have from the first equality in (24) and (44),

$$
r(x) \int K(x, y) \bar{R}(y) d y=m_{1}(x) \geq \underline{m}_{1} .
$$

The latter relations are in contradiction. Thus we have proved the

Theorem 4.1 (Non-existence of non-vanishing steady state with finite biomass) We assume (10), (4) and the second part of (9). Then there are no positive steady state to system (1) with $\varepsilon=0$.

These results suggest that steady state solutions must satisfy $\bar{n}(x)=0$ for some values of $x$; then the first equation in (24) and the above argument shows that the measure of the persistence set is small; more precisely

$$
|\{x ; \bar{n}(x)>0\}| \leq \frac{K_{1}}{\underline{m}_{1}} .
$$

When outflow rate is fast enough, species are wiped out and extinction occurs as we saw it in section 3, this is the regime $\frac{\underline{m}_{0} \underline{\underline{m}}_{2}}{r_{M}}$ large enough. But for $\frac{\underline{m}_{0} \underline{\underline{m}}_{2}}{r_{M}}$ small compared to $R_{i n}$, we may expect persistence and concentration on the fittest traits. We state later further conditions, still with finite biomass, which imply that whatever is the initial data, the solution behaves as a sum of Dirac masses.

\subsection{Dirac masses (monomorphic states)}

Indeed, as always in Lotka-Volterra equations, there are many possible Dirac steady states. Most of them are unstable and the issue of stability is studied later in section 5.3 and the present result serves as a preliminary calculation. For general coefficients $r(x), m_{1}(x)$ and $m_{2}(y)$, the monomorphic steady states are characterized in the

Theorem 4.2 (Monomorphic steady states) Consider system (1) with $\varepsilon=0$. For all $\bar{x}$ such that

$$
m_{1}(\bar{x})<r(\bar{x}) \int \frac{K(\bar{x}, y) R_{i n}(y)}{m_{2}(y)} d y
$$

there exists a unique monomorphic steady state $\bar{n}=\bar{\rho} \delta_{\bar{x}}$ with $\bar{\rho}>0$.

The biological interpretation is the following. When only one species exists (and when mutation is neglected), it can survive at a certain equilibrium density $\bar{\rho}$ if the non-extinction condition (25) is satisfied; this is in accordance with the condition for survival in section 3. Moreover, the equilibrium density is uniquely determined by the trait value of the species. 
In the particular case given in (3), where the resource-supply distribution and the competition kernel are gaussians and $m$ and $r$ are constants, the condition (25) becomes

$$
\frac{\bar{m}_{1} \bar{m}_{2}}{r}<\frac{M_{i n}}{\sqrt{2 \pi\left(\sigma_{i n}^{2}+\sigma_{K}^{2}\right)}} e^{-\bar{x}^{2} / 2\left(\sigma_{i n}^{2}+\sigma_{K}^{2}\right)} .
$$

We repeat that not all these steady states are stable under regularization of the Dirac mass or under mutations. This question will be studied later on.

Proof. Let $\bar{n}=\bar{\rho} \delta_{\bar{x}}$, with $\bar{\rho}$ to be determined later. For $\bar{n}$ to be a steady state we must have

$$
-m_{1}(\bar{x})+r(\bar{x}) \int K(\bar{x}, y) R(y, t) d y=0, \quad R(y)=\frac{R_{i n}(y)}{m_{2}(y)+r(\bar{x}) \bar{\rho} K(\bar{x}, y)} .
$$

This is equivalent to write

$$
G(\bar{\rho}, \bar{x}):=\int \frac{K(\bar{x}, y) R_{i n}(y)}{m_{2}(y)+r(\bar{x}) \bar{\rho} K(\bar{x}, y)} d y=\frac{m_{1}(\bar{x})}{r(\bar{x})} .
$$

The function $G$ is continuous and decreasing in $\bar{\rho}$. Using assumption (25) we have

$$
\lim _{\rho \rightarrow \infty} G(\rho, \bar{x})=0, \quad \lim _{\rho \rightarrow 0} G(\rho, \bar{x})=\int \frac{K(\bar{x}, y) R_{i n}(y)}{m_{2}(y)} d y>\frac{m_{1}(\bar{x})}{r(\bar{x})} .
$$

We conclude that there exists $\bar{\rho}>0$ such that $G(\bar{\rho}, \bar{x})=\frac{m_{1}(\bar{x})}{r(\bar{x})}$ and consequently $\bar{n}:=\bar{\rho} \delta_{\bar{x}}$ is a steady state.

\subsection{Dirac masses (dimorphic states)}

The problem (1) with $\varepsilon=0$ also admits polymorphic steady states. Here we consider only the case where all the functions are symmetric with respect to the origin:

$$
\begin{gathered}
R_{\text {in }}(y)=R_{\text {in }}(-y), \quad m_{1}(x)=m_{1}(-x), \quad m_{2}(y)=m_{2}(-y), \\
r(x)=r(-x), \quad K(x, y)=K(-x,-y),
\end{gathered}
$$

and we show that dimorphic steady states exist:

Theorem 4.3 (Dimorphic steady states) With the symmetry assumption (28), for all $\bar{x}$ satisfying (25) there exists a unique dimorphic steady state to system (11) with $\varepsilon=0$ in the form of

$$
\bar{n}=\bar{\rho}\left(\delta_{\bar{x}}+\delta_{-\bar{x}}\right) .
$$

We notice that the gaussian case (31) satisfies assumption (28). We deduce that, in the Gaussian case, for all $\bar{x}$ such that (26) is hold, there exists a unique $\bar{\rho}$ such that $\bar{\rho}\left(\delta_{\bar{x}}+\delta_{-\bar{x}}\right)$ is a steady state. In this case indeed both monomorphic and dimorphic steady states exist (see Section 4.2). But we insist that we do not know yet wether these states are stable under smoothing or under mutations.

Proof. We determine $\bar{\rho}$ such that $\bar{n}=\bar{\rho}\left(\delta_{\bar{x}}+\delta_{-\bar{x}}\right)$ is a steady state. We need

$$
-m_{1}(\bar{x})+r(\bar{x}) \int K(\bar{x}, y) \frac{R_{i n}(y)}{m_{2}(y)+r(\bar{x}) \bar{\rho} K(\bar{x}, y)+r(-\bar{x}) \bar{\rho} K(-\bar{x}, y)} d y=0,
$$




$$
-m_{1}(-\bar{x})+r(-\bar{x}) \int K(-\bar{x}, y) \frac{R_{\text {in }}(y)}{m_{2}(y)+r(\bar{x}) \bar{\rho} K(\bar{x}, y)+r(-\bar{x}) \bar{\rho} K(-\bar{x}, y)} d y=0 .
$$

Thanks to assumption (28), these two relations are equivalent. Therefore it is sufficient to find $\bar{\rho}$ such that the first relation holds. Following the arguments in section 4.2 , this is equivalent to write

$$
H(\bar{\rho}, \bar{x}):=\int \frac{K(\bar{x}, y) R_{\text {in }}(y)}{m_{2}(y)+\bar{\rho} r(\bar{x})[K(\bar{x}, y)+K(-\bar{x}, y)]} d y=\frac{m_{1}(\bar{x})}{r(\bar{x})} .
$$

The function $H$ is continuous and decreasing in $\bar{\rho}$. Using assumption (25) we have

$$
\lim _{\rho \rightarrow \infty} H(\rho, \bar{x})=0, \quad \lim _{\rho \rightarrow 0} H(\rho, \bar{x})=\int \frac{K(\bar{x}, y) R_{\text {in }}(y)}{m_{2}(y)} d y>\frac{m_{1}(\bar{x})}{r(\bar{x})} .
$$

We conclude that there exists a unique $\bar{\rho}>0$ such that $H(\bar{\rho}, \bar{x})=\frac{m_{1}(\bar{x})}{r(\bar{x})}$ and consequently $\bar{n}:=$ $\bar{\rho}\left(\delta_{\bar{x}}+\delta_{-\bar{x}}\right)$ is a steady state.

\section{$5 \quad$ Stability of steady states}

We continue with the case without mutations, $\varepsilon=0$. So far we have described possible steady states and proved that not all traits can be present; continuous distributions with small supports can exist as well as Dirac deltas. We now address the question of stability in these two classes. We recall the

Definition 5.1 (Evolutionary Stable Distribution, [12]) For a nonnegative bounded measure $\bar{n}$ (that is $\left.\int \bar{n}<\infty\right)$, the steady state characterized by (24) is called an Evolutionary Stable Distribution (ESD) for the equation (1) if

$$
-m_{1}(x)+r(x) \int K(x, y) \bar{R}(y) d y \leq 0, \quad \text { for all } x \in \text { Supp } n^{0} \backslash \text { Supp } \bar{n} .
$$

\subsection{Lyapunov functional and convergence to an ESD}

Here we consider a non-vanishing bounded steady state $(\bar{n}, \bar{R})$. We use the same techniques as in Section 3.3 , based on Lyapunov functionals to prove that it is nonlinearly globally attractive.

In standard analysis of adaptive dynamics, one shows the convergence and evolutionarily stability by calculating invasion fitness of rare mutants with strategy $x^{m}$ when resident population is monomorphic at strategy $x^{r}$. Our theorem extends the situation so that population consists of any (discrete or continuous) combination of strategies. The theorem strongly suggests that, if any ESD exists, any reasonable (see above) distribution of traits evolves toward the ESD. Thus, the theorem shows the convergence and evolutionarily stability of the ESD in our model against the invasion of any (discrete or continuous) combination of non-rare mutant strategies (with any densities). This is the reason we call the theorem as global convergence. In terms of the evolution of consumer trait in our chemostat model, the evolutionarily outcome is always the ESD which is determined by ecological parameters of the model (e.g., resource-supply rate, resource consumption rate) irrespective of the initial state.

Theorem 5.2 (Global convergence to ESD) Consider systems (11) or (2) with $\varepsilon=0$. We assume that $(\bar{n}, \bar{R})$ is a bounded ESD and that $\int \bar{n}(x)\left|\ln n^{0}(x)\right| d x$ and $\int \bar{R}(y)\left|\ln R^{0}(y)\right| d y$ are well-defined. Then $R(y, t) \rightarrow \bar{R}(y)$ a. e. as $t \rightarrow+\infty$. In particular this holds true if supp $n^{0}=$ Supp $\bar{n}$. 
We would like to point out that it is fundamental to assume that $\int \bar{n}(x)\left|\ln n^{0}(x)\right| d x$ is well-defined; this implies that $n^{0}$ is a function and does not vanish where $\bar{n}$ is positive (see section 5.2 for $n^{0}$ a Dirac measure). Otherwise, not only the method collapses but it is easy to build counterexamples to the result (as monomorphic cases).

Our method is closely related to the proof in [12] for direct competition. The case of discrete ESD, that is $\bar{n}$ is a measure, can be treated as well but to the expense of technical controls which are beyond the scope of the present paper.

As we will see it in the proof, convergence of $n(t)$ to $\bar{n}$ does not always follow with our mere assumptions but is true for large class of data $(r$ and $K)$. Then the uniqueness of the ESD follows and also the convergence of the population to the ESD. This shows that the systems under consideration are very particular because, in general, ESD are not always Convergence Stable Distributions (see [6, 20] and the references therein).

Proof. For system (1). With our assumptions we can define the Lyapunov functional

$$
S(t)=-\int \bar{n}(x) \ln n(x, t) d x-\int \bar{R}(y) \ln R(y, t) d y+\int n(x, t) d x+\int R(y, t) d y,
$$

and $S$ is bounded from below (because we assume that $\bar{n}$ is bounded). Using (24), we compute

$$
\frac{d S}{d t}(t)=-\int \frac{R_{\text {in }}(y)}{\bar{R}(y) R(y, t)}(\bar{R}(y)-R(y, t))^{2} d y+\int n(x, t)\left(r(x) \int K(x, y) \bar{R}(y) d y-m_{1}(x)\right) d x .
$$

Since $\operatorname{Supp} n(t, \cdot) \subseteq \operatorname{Supp} n^{0}$, assumption (29) shows that $S$ is a decreasing function. It follows that as $t \rightarrow \infty, S(t)$ converges to a finite value $\bar{S}$. We deduce that

$$
\int_{0}^{\infty} \int \frac{R_{i n}(y)}{\bar{R}(y) R(y, t)}(\bar{R}(y)-R(y, t))^{2} d y d t<+\infty .
$$

Following the arguments in the proof of Theorem 3.2 , we conclude that

$$
\lim _{t \rightarrow \infty} R(y, t)=\bar{R}(y), \quad \text { a.e. }
$$

Furthurmore, since subsequences $n\left(t_{k}\right)$ converges weakly in the sense of measures to $\widetilde{n}$, following the proof of Theorem 3.2, we have

$$
\bar{R}(y)=\frac{R_{i n}(y)}{m_{2}(y)+\int r(x) K(x, y) \widetilde{n}(x, t) d x} .
$$

This implies that for all $y, \int r(x) K(x, y) \widetilde{n}(x, t) d x=\int r(x) K(x, y) \bar{n}(x) d x$. However, our assumptions are not strong enough to conclude that $\widetilde{n}(x, t) \equiv \bar{n}(x)$; some operator invertibility is needed as in [12].

For system (2). We use the Lyapunov functional

$$
S(t)=-\int \bar{n}(x) \ln n(x, t) d x+\int n(x, t) d x
$$

It still satisfies (30) and we can apply the same method as before. 


\subsection{Population dynamics of monomorphic states}

With a variant of this method we can also study the solutions of the form $n(x, t)=\rho(t) \delta(x-\bar{x})$ corresponding to an initial data $n^{0}=\rho^{0} \delta(x-\bar{x})$; indeed such a data does not satisfy the assumptions of Theorem 5.2 because $\ln n^{0}$ is not well defined.

Theorem 5.3 For the solutions of the form $n(t)=\rho(t) \delta(x-\bar{x})$ with $\bar{x}$ satisfying (25), the population converges to the unique monomorphic steady state in Theorem 4.2. i.e. $\rho(t) \rightarrow \bar{\rho}$ and $R(y, t) \rightarrow \bar{R}(y)$ as $t \rightarrow \infty$, with $\bar{R}(y)=\frac{R_{i n}(y)}{m_{2}(y)+r(\bar{x}) \bar{\rho} K(\bar{x}, y)}$.

Proof. We treat only the case of system (1) and use the Lyapunov functional

$$
\begin{gathered}
S(t)=-\bar{\rho} \ln \rho(t)-\int \bar{R}(y) \ln (R(y, t)) d y+\rho(t)+\int R(y, t) d y, \\
\frac{d S}{d t}(t)=-\int \frac{R_{\text {in }}(y)}{\bar{R}(y) R(y, t)}(\bar{R}(y)-R(y, t))^{2} d y .
\end{gathered}
$$

With similar arguments as above we obtain that $R(y, t) \underset{t \rightarrow \infty}{\longrightarrow} \bar{R}(y)$. Since there exists a unique constant $\bar{\rho}$ such that $\bar{R}(y)=\frac{R_{i n}(y)}{m_{2}(y)+r(\bar{x}) \bar{\rho} K(\bar{x}, y)}$, following again the arguments in the proof of Theorem 5.2 we obtain that $\rho(t) \underset{t \rightarrow 0}{\longrightarrow} \bar{\rho}$.

\subsection{Condition for a monomorphic state to be an ESD}

With the extension in [12], we may expect that Theorem 5.2 applies in particular for monomorphic steady states. We consider such a monomorphic distribution $\bar{n}=\bar{\rho} \delta(x)$, restricting ourselves to a population concentrated in the origin and give a condition implying that it is evolutionary stable. Here, to simplify the analysis, we consider only the 'gaussian' case given in (3).

Along with section 4.2, we need the non-extinction condition (26) with $\bar{x}=0$, that is

$$
\frac{\bar{m}_{1} \bar{m}_{2}}{r M_{i n}} \sqrt{2 \pi}<\frac{1}{\sqrt{\sigma_{i n}^{2}+\sigma_{K}^{2}}} .
$$

To check that the ESD condition holds locally, it is enough to check the sign of the second derivative of the fitness function (see Appendix A). The exact condition is given by formula (60) which is not tractable. That is why we compute an easier sufficient condition given by the

Theorem 5.4 (Sufficient condition for ESD) Consider the gaussian case (3) with the condition (32). Let $n=\bar{\rho} \delta$ with $\bar{\rho}$ as in (27) be a steady state. We additionally assume that

$$
\frac{\sigma_{i n}^{2}}{\left(\sigma_{i n}^{2}+\sigma_{K}^{2}\right)^{\frac{3}{2}}}<\frac{\bar{m}_{1} \bar{m}_{2}}{r M_{i n}} \sqrt{2 \pi} .
$$

Then the monomorphic state $n=\bar{\rho} \delta_{0}$ is a local ESD to system (11) with $\varepsilon=0$, in other words for Supp $n^{0} \supset\{0\}$ small enough then (29) holds. 
This Theorem involves technical calculations and we prove it in Appendix A.

In conclusion for $n=\bar{\rho} \delta_{0}$ to be an ESD, using (32), it is enough that

$$
\frac{\sigma_{i n}^{2}}{\left(\sigma_{i n}^{2}+\sigma_{K}^{2}\right)^{\frac{3}{2}}}<\frac{\bar{m}_{1} \bar{m}_{2}}{r M_{i n}} \sqrt{2 \pi}<\frac{1}{\sqrt{\sigma_{i n}^{2}+\sigma_{K}^{2}}} .
$$

Roughly, it means that $\sigma_{K}$ is large enough compared to $\sigma_{i n}$. In biological words, when the outflow rate is not too big and the resource supply distribution has a sharper peak than the competition kernel, then the species distribution is isolated.

The condition (34) is not a necessary condition for an ESD. We give an example where the condition is not satisfied but the origin is nevertheless an ESD. We consider the following parameter values

$$
\sigma_{K}=1, \quad \sigma_{i n}=1, \quad \bar{m}_{1}=0.5, \quad \bar{m}_{2}=0.5, \quad M_{\text {in }}=3, \quad r=1 .
$$

We can easily verify that the condition (34) is not satisfied with these parameters. However as we see in Figure 3 the population goes to the origin and remains there. This is due to the estimations that we have used in our calculations. In this particular case we can compute numerically the second derivative of the fitness function using (60) in Appendix A

$$
D^{2} F_{0}(0)=-\frac{\bar{m}_{1}}{\sigma_{K}^{2}}+\frac{r M_{i n}}{2 \pi \sigma_{K}^{5} \sigma_{i n}} \int x^{2} \frac{e^{\frac{-x^{2}}{2}\left(\frac{1}{\sigma_{K}^{2}}+\frac{1}{\sigma_{i n}^{2}}\right)}}{\bar{m}_{2}+\bar{\rho} K(-x)} d x \approx-0.16,
$$

where we estimate numerically $\bar{\rho} \approx 3.9$. This confirms that the origin is an ESD.

If we decrease $\sigma_{K}$ to 0.5 the origin is not ESD anymore and we observe a branching. In this case not only the condition (34) is satisfied but the exact amount of $D^{2} F_{0}(0) \approx 0.59$ is also positive and we estimate numerically $\bar{\rho} \approx 3.2$. This is in accordance with numerical results in Figure 3 ,
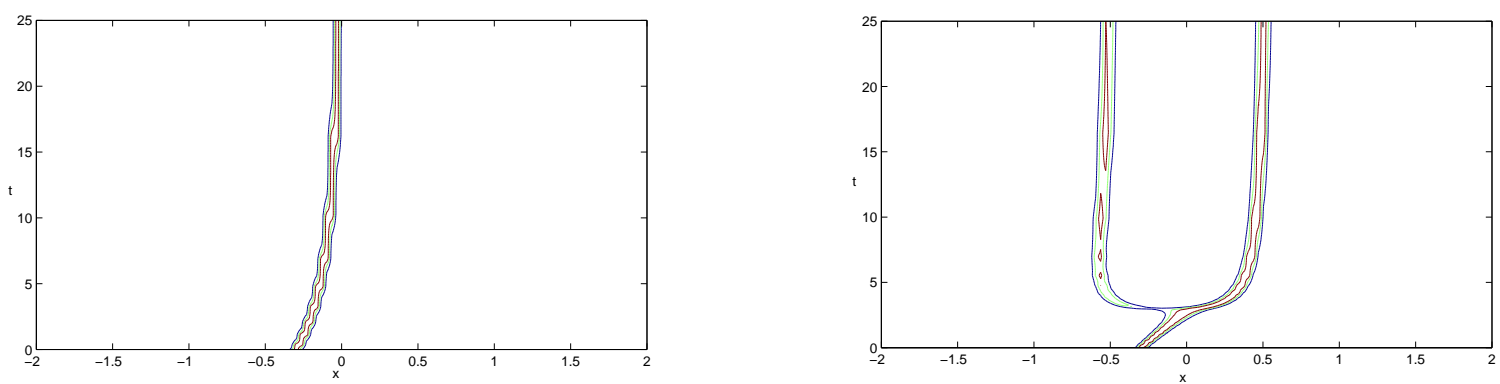

Figure 3: Dynamics of dominant traits begining with an initial data concentrated in -0.3 . We present the dynamics of the model given in (2) in the particular case of (3) with $\varepsilon=0.001, \sigma_{i n}=1, r=1$, $\bar{m}_{1}=\bar{m}_{2}=0.5, M_{i n}=3$. Left $\sigma_{K}=1$. Right $\sigma_{K}=.5$. We observe that for $\sigma_{i n}$ large comparing to $\sigma_{K}$ the monomorphic case is no more evolutionary attractor and we observe branching.

\section{Dynamics of the fittest traits: an asymptotic point of view}

We have shown that the model has monomorphic or polymorphic steady states and derived the sufficient condition for a monomorphic distribution (Dirac mass) to be evolutionarily stable. To obtain a stronger result than merely local stability, here we consider a case when a population is initially 
concentrated around one or several points in trait space. We establish that it stays concentrated and, using asymptotic analysis, that the fittest traits follow a form of canonical equation. We also give conditions implying that the population becomes monomorphic.

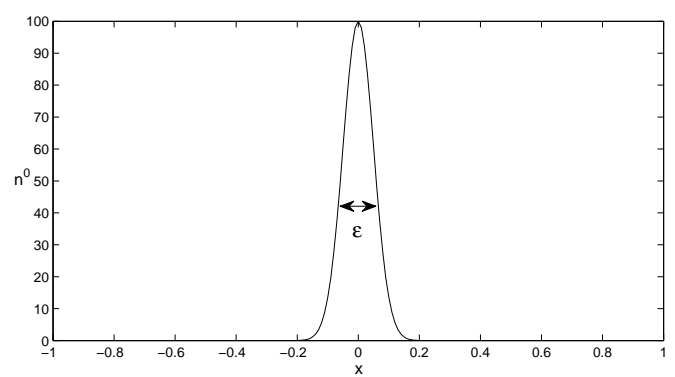

Figure 4: The parameter $\varepsilon$ in (36) measures how close is the distribution from the Dirac distribution, possibly due to mutations or, as considered here, initial variations.

We are interested in the dynamics with an initial data 'close' to a monomorphic or polymorphic state

$$
n_{\varepsilon}(x, 0) \approx \sum_{i=1, \ldots, I} \bar{\rho}_{i}^{0} \delta\left(x-\bar{x}_{i}^{0}\right)
$$

To track the movements of $\bar{x}_{i}$ (i.e., evolutionary dynamics of traits), the initial data are chosen as a sum of concentrations with tails (non-zero variances in trait values). A parameter $\varepsilon$ denotes a small deviation of the initial data from the corresponding strictly monomorphic/polymorphic states (Dirac masses), see Figure 4. We also perform a change of variable $\tau=\varepsilon t$ in order to accelerate time and observe the dynamics. Note that the scaling parameter $\varepsilon$ here is no longer a mutation rate. Mathematically, however, the existence of continuously positive distribution is sufficient to track evolutionary dynamics. With this new time, the quasi-static model becomes

$$
\left\{\begin{array}{l}
\frac{\partial}{\partial \tau} n_{\varepsilon}=\frac{n_{\varepsilon}}{\varepsilon}\left(-m_{1}(x)+r(x) \int K(x, y) R_{\varepsilon}(y, \tau) d y\right), \\
R_{\varepsilon}(y, \tau)=\frac{R_{i n}(y)}{m_{2}(y)+\int r(x) K(x, y) n(x, \tau) d x} .
\end{array}\right.
$$

Starting from these approximately monomorphic/polymorphic populations, we show that under some assumptions the number of concentrated peaks does not change in time and no evolutionary branching can happen. However the dominant traits can evolve in time and we can describe the motion of these dominant traits with a Hamilton-Jacobi equation. We also obtain a minimum distance between the dominant traits. In section 6.2 we give a form of a canonical equation of the dominant traits. These results correspond to the convergence stability in Adaptive Dynamics, and the biological implications are discussed in section 6.3.

\subsection{Polymorophic dynamics: mathematical results}

According to the usual approximation of Dirac masses by gaussians, we describe it by the properties of the population potential $\varphi_{\varepsilon}^{0}(x)$ in

$$
n_{\varepsilon}(x, 0)=e^{\frac{\varphi_{\varepsilon}^{0}(x)}{\varepsilon}} .
$$


The theory developed from [7] for the Lotka-Volterra equations shows that the representation $\varphi_{\varepsilon}(x, t):=$ $\varepsilon \ln \left(n_{\varepsilon}(x, t)\right)$ makes sense for all times because it satisfies the simple equation

$$
\frac{\partial}{\partial \tau} \varphi_{\varepsilon}(x, \tau)=-m_{1}(x)+r(x) \int K(x, y) R_{\varepsilon}(y, \tau) d y .
$$

Of course there is a shortcoming in this point of view because the scaling parameter $\varepsilon>0$ is defined through the initial data which seems arbitrary. The real motivation is from modeling of mutations which we know induces this type of smoothing of the population density. See [7, 1, 18, 2, It has been widely proved that the method may handle these mutations as well in self-contained population models as (11), (2).

Our main characterization of the population density goes through the function $\varphi_{\varepsilon}$ and we assume that initially

$$
\left|\varphi_{\varepsilon}^{0}(x)\right| \leq C_{0}(R), \quad\left|\nabla \varphi_{\varepsilon}^{0}(x)\right| \leq C_{1}(R), \quad \forall|x| \leq R .
$$

As a preliminary result, we prove in Appendix $\mathrm{C}$ the following

Proposition 6.1 (Convergence) With the assumptions (44), (6), (99), (8), (111) and (38), after successive extractions of subsequences, the family $\varphi_{\varepsilon}$ converges locally uniformly to a continuous function $\varphi \leq 0$, the family $R_{\varepsilon}$ converges locally uniformly in space and weakly in time to a limit $R(y, \tau)$. Furthermore, we have the relation

$$
\varphi(x, \tau)=\varphi^{0}(x)-m_{1}(x) \tau+r(x) \int_{0}^{\tau} \int K(x, y) R(y, s) d y d s, \quad \varphi(x, \tau) \leq 0 .
$$

With these assumptions, the conclusion of Theorem 3.1 holds and we may extract a subsequence such that the $n_{\varepsilon}$ 's converge weakly in the sense of measures to a limit $n(x, \tau)$. The support of $n$ is included in the set $\{(\tau, x) \mid \varphi(x, \tau)=0\}$ (see [7, 18, 4, 14] for several uses of this property). We show below that the zeros of $\varphi(\tau)$ are isolated points, and thus the model leads to Dirac concentrations.

Theorem 6.2 (Concentration effects) We assume that, for some positive constant $a_{0}$ and $\mu_{1} \in \mathbb{R}$,

$$
\varphi_{\varepsilon, x x}^{0}(x)-\mu_{1} \varphi_{\varepsilon}^{0}(x) \leq a_{0},
$$

(i) and that there is $a_{1}>0$ and $\mu_{2} \geq 0$ such that

$$
m_{1}^{\prime \prime}(x)-m_{1}(x) \mu_{1}-\int \frac{\mu_{2}(y)}{m_{2}(y)} R_{i n}(y) d y \geq a_{1}, \quad(r(x) K(x, y))_{x x} \leq \mu_{1} r(x) K(x, y)+\mu_{2}(y) .
$$

Then we have

$$
\varphi_{x x}-\mu_{1} \varphi \leq a_{0}-a_{1} \tau
$$

in the distributional sense and a.e. In particular if $a_{0} \leq 0$, then we have $\varphi_{x x}-\mu_{1} \varphi \leq 0$ and thus, after extraction of a subsequence, the $n_{\varepsilon}$ 's converge in the weak sense of measures to a sum of Dirac masses:

$$
n_{\varepsilon}(x, \tau) \rightarrow n(x, \tau)=\sum_{i} \bar{\rho}_{i}(\tau) \delta_{i}\left(x-\bar{x}_{i}(\tau)\right) .
$$

(ii) If (41) is hold with $\mu_{1} \geq 0$ then, the population, when it persists, is asymptotically monomorphic:

$$
n_{\varepsilon}(x, \tau) \rightarrow n(x, \tau)=\bar{\rho}(\tau) \delta(x-\bar{x}(\tau)),
$$


and the pair $(\bar{x}(\tau), \bar{\rho}(\tau))$ also satisfies

$$
\bar{\rho}(\tau)\left(-m_{1}(\bar{x}(\tau))+r(\bar{x}(\tau)) \int K(\bar{x}(\tau), y) R(y, \tau) d y\right) \geq 0 .
$$

Also, the equation for $R(y, \tau)$ can be reduced to

$$
\begin{gathered}
R(y, \tau)=\frac{R_{i n}(y)}{m_{2}(y)+\sum_{i} \bar{\rho}_{i}(\tau) r\left(\bar{x}_{i}(\tau)\right) K\left(\bar{x}_{i}(\tau), y\right)} \quad \text { (polymorphic case) } \\
R(y, \tau)=\frac{R_{i n}(y)}{m_{2}(y)+\bar{\rho}(\tau) r(\bar{x}(\tau)) K(\bar{x}(\tau), y)} \quad \text { (monomorphic case) }
\end{gathered}
$$

In order to illustrate our assumptions, we may consider the case when

$$
\begin{gathered}
r(x)=r_{0}, \quad m_{1}(x)=\widetilde{m}_{0}+\widetilde{m}_{1} \frac{|x|^{2}}{2}, \quad \widetilde{m}_{2}(y) \geq m_{2}, \\
R_{i n}=\frac{M_{i n}}{\sigma_{i n} \sqrt{2 \pi}} \exp \left(-\frac{y^{2}}{2 \sigma_{i n}^{2}}\right), \quad K(z)=\frac{1}{\sigma_{K} \sqrt{2 \pi}} \exp \left(-\frac{z^{2}}{2 \sigma_{K}^{2}}\right)
\end{gathered}
$$

and $K(x, y)=K(x-y)$. We compute

$$
K_{x x}=\left[-\frac{1}{\sigma_{K}^{2}}+\frac{|z|^{2}}{\sigma_{K}^{4}}\right] K, \quad \mu_{1}=0, \quad \mu_{2}=\frac{C}{\sigma_{K}^{3}} .
$$

Therefore $\widetilde{m}_{1} \widetilde{m}_{2}$ large enough compared to $\frac{M_{i n}}{\sigma_{K}^{3}}$ implies monomorphism.

We can also take $\widetilde{m}_{1}=0$, i.e., $m_{1}$ constant, $\mu_{1}=-\frac{1}{\sigma_{K}^{2}}$. Then, $\frac{\widetilde{m}_{0}}{\sigma_{K}^{2}}$ large enough compared to $\frac{M_{i n}}{\sigma_{K}^{3} \widetilde{m}_{2}}$ implies polymorphism but not continuous distribution.

Proof. We differentiate twice equation (37) and using (41) we obtain

$$
\begin{aligned}
\frac{\partial}{\partial \tau} \varphi_{\varepsilon, x x} & =-m_{1}^{\prime \prime}(x)+\int(r(x) K(x, y))_{x x} R_{\varepsilon}(y, \tau) d y \\
& \leq-m_{1}^{\prime \prime}(x)+\mu_{1} \int r(x) K(x, y) R_{\varepsilon}(y, \tau) d y+\int \mu_{2}(y) R_{\varepsilon}(y, \tau) d y \\
& \leq-m_{1}^{\prime \prime}(x)+\mu_{1} m_{1}(x)+\mu_{1} \frac{\partial}{\partial \tau} \varphi_{\varepsilon}(x, \tau)+\int \frac{\mu_{2}(y)}{m_{2}(y)} R_{i n}(y) d y
\end{aligned}
$$

We deduce that, using again (41),

$$
\frac{\partial}{\partial \tau}\left(\varphi_{x x}-\mu_{1} \varphi\right) \leq-m_{1}^{\prime \prime}(x)+\mu_{1} m_{1}(x)+\int \frac{\mu_{2}(y)}{m_{2}(y)} R_{i n}(y) d y \leq-a_{1}
$$

Therefore, using (40), we have

$$
\varphi_{x x}-\mu_{1} \varphi \leq a_{0}-a_{1} \tau \text {. }
$$

In particular, if $a_{0} \leq 0$, we deduce that $\varphi_{x x}<0$ in the set $\{(t, x) \mid \varphi(\tau, x)=0\}$. Therefore the zeros of $\varphi$ are isolated. Since the support of $n$ is included in this set, we conclude that $n$ is a sum of Dirac masses as stated in (43). 
Furthermore if $\mu_{1} \geq 0$ then we deduce that $\varphi \leq 0$ is a strictly concave function with respect to $x$. Thus it has a unique maximum. We conclude that the population, if it does not go extinct, is monomorphic as stated in (44).

Finally, we integrate (35) and obtain

$$
\varepsilon \frac{d}{d \tau} \bar{\rho}(\tau)=-\int n_{\varepsilon}(\tau, x) m_{1}(x) d x+\iint n_{\varepsilon}(\tau, x) r(x) K(x, y) \frac{R_{\text {in }}(y)}{m_{2}(y)+\int r\left(x^{\prime}\right) K\left(x^{\prime}, y\right) n_{\varepsilon}\left(\tau, x^{\prime}\right) d x^{\prime}} d y d x .
$$

Passing to the weak limit we obtain that

$$
\begin{aligned}
0 & =-\bar{\rho}(\tau) m_{1}(\bar{x}(\tau))+\mathrm{w}-\lim \iint n_{\varepsilon}(\tau, x) r(x) K(x, y) \frac{R_{\text {in }}(y)}{m_{2}(y)+\int r\left(x^{\prime}\right) K\left(x^{\prime}, y\right) n_{\varepsilon}\left(\tau, x^{\prime}\right) d x^{\prime}} d y d x \\
& \leq \bar{\rho}(\tau)\left(-m_{1}(\bar{x}(\tau))+\int r(\bar{x}(\tau)) K(\bar{x}(\tau), y) \frac{R_{i n}(y)}{m_{2}(y)+\bar{\rho}(\tau) r(\bar{x}(\tau)) K(\bar{x}(\tau), y)} d y\right) .
\end{aligned}
$$

Hence (45).

\subsection{A form of canonical equation}

Within the framework of Section 6.1 we can go further and obtain several informations on the dynamics of the fittest trait, including a form of canonical equation as proposed initially in [5]. For this, we need some additional regularity which relies on the assumptions

$$
\left|D^{3} \varphi^{0}(x)\right| \leq D_{1}, \quad\left|D^{3} m(x)\right| \leq D_{2}, \quad\left|D_{x}^{3} K(x, y)\right| \leq D_{3}, \quad\left|D^{3} r(x)\right| \leq D_{4} .
$$

This implies that $\varphi$ and $R$ also have bounded third derivatives in $x$. We also need the following additional assumption on the initial data

$$
-m_{1}(\bar{x}(0))+r(\bar{x}(0)) \int K(\bar{x}(0), y) \frac{R_{i n}(y)}{m_{2}(y)} d y>0 .
$$

Within the framework of Theorem 6.2 and in the monomorphic case we prove the following

Theorem 6.3 (Form of canonical equation) We assume (48), (49) and (41) with $\mu_{1} \geq 0$, (40) with $a_{0} \leq 0$ and thus (44). Then, $\bar{x} \in W^{1, \infty}$ and the following form of canonical equation for the fittest trait holds

$$
\begin{gathered}
\dot{\bar{x}}(\tau)=\left(-D^{2} \varphi(\bar{x}(\tau), \tau)\right)^{-1} \quad\left(-\nabla m_{1}(\bar{x}(\tau))+r(\bar{x}(\tau)) \int \nabla_{x} K(\bar{x}(\tau), y) R(y, \tau) d y\right. \\
\left.+\nabla r(\bar{x}(\tau)) \int K(\bar{x}(\tau), y) R(y, \tau) d y\right) .
\end{gathered}
$$

Moreover, the family $\rho_{\varepsilon}$ converges a.e. to $\bar{\rho} \in W^{1, \infty}$, and we have

$$
\begin{gathered}
-m_{1}(\bar{x}(\tau))+\int K(\bar{x}(\tau), y) R(y, \tau) d y=0, \quad R(y, \tau)=\frac{R_{i n}(y)}{m_{2}(y)+\bar{\rho}(\tau) r(\bar{x}(\tau)) K(\bar{x}(\tau), y)}, \\
\bar{\rho}(t) \geq \rho^{0} e^{-K \tau} .
\end{gathered}
$$

In particular, we deduce that the population does not get extinct. 
This Theorem is proved in Appendix D.

We notice that for any $(\tau, \bar{x}(\tau))$ there exists a unique $\bar{\rho}(\tau)$ such that (151) is satisfied. Therefore by eliminating $\bar{\rho}(\tau)$ from the canonical equation (50) we obtain an equation that depends only on the parameter $\bar{x}(\tau)$. However, we cannot write the canonical equation explicitly because the formula for $\bar{\rho}(\tau)$ is implicit.

We may also write canonical equations for dominant traits for the case with polymorphic populations within the framework of Section 6.1 when there are only a finite number of traits. We obtain the set of constraints and, with $R(y, t)$ given in (46), the canonical equation

$$
\begin{aligned}
\dot{\bar{x}}_{i}(\tau)=\left(-D^{2} \varphi\left(\bar{x}_{i}(\tau), \tau\right)\right)^{-1}(- & m_{1}\left(\bar{x}_{i}(\tau)\right)+r\left(\bar{x}_{i}(\tau)\right) \int \nabla_{x} K\left(\bar{x}_{i}(\tau), y\right) R(y, \tau) d y \\
& \left.+\nabla r\left(\bar{x}_{i}(\tau)\right) \int K\left(\bar{x}_{i}(\tau), y\right) R(y, \tau) d y\right) .
\end{aligned}
$$

\subsection{Biological implications: Generation of species}

We now give the biological implications of our mathematical result and focus on the following two points.

(i) The interpretation of the trait concentration in terms of the function $\varphi$ is

$$
n_{\varepsilon}(x, \tau) \approx \sum_{i=1, \ldots, I} \rho_{i}(\tau) \delta\left(x-\bar{x}_{i}(\tau)\right) \Longleftrightarrow \max _{x} \varphi(x, \tau)=0=\varphi\left(\bar{x}_{i}(\tau), \tau\right) .
$$

But at the points such that $\varphi\left(\bar{x}_{i}(\tau), \tau\right)=0$ for $\tau>\tau_{0}:=\frac{a_{0}}{a_{1}}$, using Theorem 6.2, we have

$$
\varphi_{x x}\left(\bar{x}_{i}(\tau), \tau\right) \leq-a_{1}\left(\tau-\tau_{0}\right)<0 .
$$

We deduce that for $\tau>\tau_{0}$, the zeros of $\varphi$ are isolated. Therefore $n(\tau, x)$ is in the form of a sum of Dirac masses after $\tau>\tau_{0}$ (to be rigorous we need to complement our assumptions to ensure that $\varphi$ has three derivatives in $x$; this follows when data have three derivatives).

(ii) We can also give a minimal distance between two Dirac masses after time $\tau_{0}$, using the uniform Lipschitz bound on $\varphi$, see Appendix C, Let $\varphi\left(\tau, x_{i}(\tau)\right)=0$. We have

$$
\begin{aligned}
\varphi_{x x}(\tau, x) & \leq a_{0}-a_{1} \tau+\mu_{1} \varphi(\tau, x) \\
& \leq a_{0}-a_{1} \tau+\frac{\left(C_{1}+B_{1} \tau\right)}{\mu_{1}}\left|x-x_{i}(\tau)\right| .
\end{aligned}
$$

We deduce that $\varphi_{x x}(\tau, x)<0$ for

$$
\left|x-x_{i}(\tau)\right|<\frac{a_{1} \tau-a_{0}}{B_{1} \tau+C_{1}} .
$$

Therefore the minimal distance between two Dirac masses at time $t$ is

$$
\left|x_{i}(\tau)-x_{i+1}(\tau)\right| \leq 2 \frac{a_{1} \tau-a_{0}}{B_{1} \tau+C_{1}} .
$$

Biologically, these two results mean that distinct species remain distinct after the finite time $\tau_{0}$. We can not observe any continuous branching or merging for $\tau>\tau_{0}$, because at the point where branching 
(or merging) happens the two new (or previous) Dirac masses will be (have been) very near each other for a while. But according to (ii) this cannot happen. Thus, the number of species does not increase (but can decrease because extinction is still possible) after this threshold time (although their trait values might keep moving). Evolutionary diversification (generation of new species) must finish until this time, so $\tau_{0}$ can be considered as a time scale of evolution.

Note that $\tau_{0}$ is measured in a slower time-scale than $t$ in the full model. This is necessary as we take $\varepsilon \rightarrow 0$, corresponding to no mutation limit, otherwise evolution requires infinitely long time. Interestingly, we still observe the dynamics in finite time in this evolutionary time scale. What is unique to the present study is that we have shown the finite threshold time after which no structual change occurs in evolutionary dynamics. This is a non-trivial result because any quantity (especially selection gradient) does not depend explicitly on time except initial data, that are chosen infinitesimally close to Dirac masses.

In some individual-based simulations of evolution of species traits (see [11]), we observe endlesslyrepeated extinctions and generations of species, i.e. recurrent adaptive radiations. This has been considered as a stochastic effect, but in principle this could be possible in deterministic system as well. Our result shows that such recurrent adaptive radiation never occurs in our system.

\section{Transient from continuous distribution to Dirac mass}

Here we extand the analysis in section 6 to more general initial distribution. We give details on how an initially continuous population becomes approximately monomorphic/polymorphic in large time. Once this stage is reached, all the results and biological implications in the previous section are valid. Hence, this section is a natural extension of our results to relax our assumption of approximately monomorphic/polymorphic initial data.

We come back to the quasi-static model

$$
\left\{\begin{array}{l}
\frac{\partial n(x, t)}{\partial t}=n(x, t)\left(-m_{1}(x)+r(x) \int K(x, y) R(y, t) d y\right) \\
R(y, t)=\frac{R_{i n}(y)}{m_{2}(y)+\int r\left(x^{\prime}\right) K\left(x^{\prime}, y\right) n\left(x^{\prime}, t\right) d x^{\prime}} .
\end{array}\right.
$$

We aim to show that for large times, the population density behaves like a highly concentrated gaussian around an unknow fittest trait $\bar{x}(t)$, by example

$$
n(x, t) \approx \frac{\bar{\rho}(t)}{\sqrt{2 \pi t}} \exp \left(-\frac{|x-\bar{x}(t)|^{2}}{2 t}\right) .
$$

The model does not impose such a quadratic type of concentration to a Dirac mass and we have to generalize the approximation as

$$
n(x, t) \approx e^{\varphi(x, t)}, \quad \text { with } \varphi \text { a uniformly concave function in } x .
$$

Then $\bar{x}(t)$ is the point where $\max _{x} \varphi(x, t)=\varphi(\bar{x}(t), t)$.

\subsection{Pointwise estimate on the population density}

We continue our analysis and prove an upper bound on pointwise growth of the population. To do so, we use the population potential

$$
\varphi(x, t)=\ln (n(x, t)) .
$$


Here, as in (38), we use the following assumptions initially

$$
\varphi(x, 0) \leq C_{0}, \quad\left|\varphi_{x}(x, 0)\right| \leq C_{1}, \quad\left|\varphi_{x x}(x, 0)\right| \leq C_{2} .
$$

Proposition 7.1 (Pointwise esimate) Under assumptions (41), (9), (5), (58) and the first parts of (6) and (11), there is a constant $A>0$ (depending on $\underline{m}_{2}, M_{1}, r_{M}, r_{0}, r_{2}, K_{2}, K_{M}, M_{i n}, C_{0}, C_{1}$ ) such that at all points $x$,

$$
n(x, t) \leq(2+t)^{A}, \quad \varphi(x, t) \leq A \ln (2+t) .
$$

It turns out that polynomial growth on $n(t)$ is indeed true under some additional assumption that we mention later. This is a first indication that concentration on the population should indeed occur.

This Theorem is proved in Appendix E.

\subsection{Towards monomorphism or polymorphism}

We are now ready to establish the result showing strong convergence to a monomorphic or polymorphic population.

Theorem 7.2 (Sufficient condition for convergence to well-separated traits) With the above assumptions (5) -(11), (58) and

(i) If (41) holds with $\mu_{1} \geq 0$, then $\varphi$ is concave for $t$ large enough and the population, if it persists, is asymptotically monomorphic, namely

$$
\limsup _{t \rightarrow \infty} \frac{\varphi_{x x}(x, t)}{t} \leq-a_{1}
$$

(ii) If (41) holds with $\mu_{1}<0$, then

$$
\limsup _{t \rightarrow \infty} \frac{\varphi_{x x}(x, t)-\mu_{1} \varphi(x, t)}{t} \leq-a_{1} .
$$

Consequently, the population is concentrated on well separated traits and the distribution cannot be continuous.

The unique maximum point $\bar{x}(t)$ of $\varphi(\cdot, t)$ defines the fittest (highest represented) trait. It might be that $\bar{x}(t)$ escapes to infinity for large times, as in the cannibalism model (see [6, 17] for instance).

Proof. We write

$$
\frac{\partial}{\partial t} \varphi(x, t)=-m_{1}(x)+r(x) \int K(x, y) R(y, t) d y .
$$

We differentiate twice this equation and, using (41), we find

$$
\begin{aligned}
\frac{\partial}{\partial t} \varphi_{x x}(x, t) & =-m_{1}^{\prime \prime}(x)+\int(r(x) K(x, y))_{x x} R(y, t) d y \\
& \leq-m_{1}^{\prime \prime}(x)+\mu_{1} \int r(x) K(x, y) R(y, t) d y+\int \mu_{2}(y) R(y, t) d y \\
& \leq-m_{1}^{\prime \prime}(x)+\mu_{1} m_{1}(x)+\mu_{1} \frac{\partial}{\partial t} \varphi(x, t)+\int \frac{\mu_{2}(y)}{m_{2}(y)} R_{i n}(y) d y \\
& \leq-a_{1}+\mu_{1} \frac{\partial}{\partial t} \varphi(x, t)
\end{aligned}
$$


Integrating in time, we obtain

$$
\varphi_{x x}(x, t) \leq \varphi_{x x}^{0}(x)-a_{1} t+\mu_{1}\left[\varphi(t, x)-\varphi^{0}(x)\right] .
$$

With the help of assumptions (58) and Theorem 7.1, we conclude the first case (monomorphism).

In the case $\mu_{1}<0$, we use the same computation and arrive at

$$
\frac{\partial}{\partial t}\left[\varphi_{x x}(x, t)-\mu_{1} \varphi(x, t)\right] \leq-m_{1}^{\prime \prime}(x)+\mu_{1} m_{1}(x)+\int \frac{\mu_{2}(y)}{m_{2}(y)} R_{i n}(y) d y \leq-a_{1} .
$$

The conclusion follows as before.

Acknowledgment The three authors thank Readilab, LIA 197 CNRS, for supporting their collaborations and also Meiji University and UPMC. J.Y.W. is partly supported by Glocal COE program "Formation and Development of Mathematical Sciences Based on Modeling and Analysis".

\section{A Proof of Theorem 5.4}

With the terminology in [6, 19], we define the fitness function as

$$
F_{0}(x)=-\bar{m}_{1}+r \int K(x-z) \frac{R_{\text {in }}(z)}{\bar{m}_{2}+r \bar{\rho} K(-z)} d z .
$$

Ii measures the invasion ability of a mutant $x$ when the residents are all at the origin. For an ESD concentrated at the origin, the fitness function must satisfy $D F_{0}(0)=F_{0}(0)=0$ and $D^{2} F_{0}(0)<0$.

As we computed it in section 4.2 the condition $F_{0}(0)=0$ follows from the choice of the weight $\bar{\rho}$ of the Dirac mass $\bar{n}=\bar{\rho} \delta$, which can also be written

$$
\bar{m}_{1}=r K * R(0)=r \int K(y) \frac{R_{i n}(y)}{\bar{m}_{2}+r \bar{\rho} K(y)} d y .
$$

We also have, since $D K$ is odd and $K$ and $R_{\text {in }}$ are even,

$$
D F(0)=r \int D K(-y) \frac{R_{i n}(y)}{\bar{m}_{2}+r \bar{\rho} K(-y)} d y=0 .
$$

Finally, we analyse the sign of

$$
D^{2} F=r D^{2} K * R .
$$

Next, we perform some preliminary calculation

$$
\begin{gathered}
\bar{m}_{1}=\frac{r M_{i n}}{2 \pi \sigma_{K} \sigma_{i n}} \int \frac{e^{\frac{-x^{2}}{2}\left(\frac{1}{\sigma_{K}^{2}}+\frac{1}{\sigma_{i n}^{2}}\right)}}{\bar{m}_{2}+\bar{\rho} K(x)} d x, \\
D^{2} K(x)=\frac{1}{\sigma_{R}^{3} \sqrt{2 \pi}}\left(-1+\frac{x^{2}}{\sigma_{R}^{2}}\right) \exp \left(-\frac{x^{2}}{2 \sigma_{R}^{2}}\right) .
\end{gathered}
$$

Therefore, the condition for a local ESD is

$$
D^{2} F_{0}(0)=-\frac{\bar{m}_{1}}{\sigma_{K}^{2}}+\frac{r M_{i n}}{2 \pi \sigma_{K}^{5} \sigma_{i n}} \int x^{2} \frac{e^{\frac{-x^{2}}{2}\left(\frac{1}{\sigma_{K}^{2}}+\frac{1}{\sigma_{i n}^{2}}\right)}}{\bar{m}_{2}+\bar{\rho} K(-x)} d x<0 .
$$


Define $\sigma^{\prime}=\frac{\sigma_{K} \sigma_{i n}}{\sqrt{\sigma_{K}^{2}+\sigma_{i n}^{2}}}$. We have

$$
\begin{aligned}
D^{2} F_{0}(0) & \leq-\frac{\bar{m}_{1}}{\sigma_{K}^{2}}+\frac{r M_{i n}}{2 \pi \sigma_{K}^{5} \sigma_{i n} \bar{m}_{2}} \int x^{2} e^{\frac{-x^{2}}{2 \sigma^{\prime 2}}} d x \\
& =-\frac{\bar{m}_{1}}{\sigma_{K}^{2}}+\frac{r M_{i n} \sigma^{\prime 3}}{\sqrt{2 \pi} \sigma_{K}^{5} \sigma_{i n} \bar{m}_{2}} \\
& =-\frac{\bar{m}_{1}}{\sigma_{K}^{2}}+\frac{r M_{i n}}{\sqrt{2 \pi} \sigma_{K}^{2} \bar{m}_{2}} \cdot \frac{\sigma_{i n}^{2}}{\left(\sigma_{i n}^{2}+\sigma_{K}^{2}\right)^{\frac{3}{2}}}
\end{aligned}
$$

We deduce from this inequality that $D^{2} F_{0}(0)<0$ under the condition (33). This condition is however not sharp because we neglected the helping term $\bar{\rho} K(-x)$.

\section{B Lipschitz bounds on $\varphi$}

Several steps in our estimates use Lipschitz bounds on the function $\varphi$ or $\varphi_{\varepsilon}$ defined through the logarithmic transform.

First we show that $|\nabla \varphi|(x, t) \leq C_{1}+B_{1} t$, for all $x$, and for $B_{1}$ a constant that we determine later. We replace $n=e^{\varphi}$ in (56) and find

$$
\frac{\partial}{\partial t} \varphi=-m_{1}(x)+r(x) \int K(x, y) R(y, t) d y .
$$

Differentiating this equation we obtain

$$
\frac{\partial}{\partial t} \varphi_{x}=-m_{1, x}(x)+r(x) \int K_{x}(x, y) R(y, t) d y+r_{x}(x) \int K(x, y) R(y, t) d y .
$$

Using (4), (9), (6) , the second part of (5) and the first part of (11) we have

$$
\left|\frac{\partial}{\partial t} \varphi_{x}\right| \leq M_{1}+\frac{r_{M} K_{2} M_{i n}}{\underline{m}_{2}}+\frac{r_{2} K_{M} M_{i n}}{\underline{m}_{2}} .
$$

From this and (58) we deduce that

$$
\left|\varphi_{x}(x, t)\right| \leq C_{1}+B_{1} t, \quad B_{1}=M_{1}+\frac{r_{M} K_{2} M_{i n}}{\underline{m}_{2}}+\frac{r_{2} K_{M} M_{i n}}{\underline{m}_{2}} .
$$

\section{Proof of Theorem 6.1}

(i) (Lipschitz bound in space for $\varphi_{\varepsilon}$ ) We recall the inequality (62) proved earlier $\left|\nabla \varphi_{\varepsilon}(x, \tau)\right| \leq$ $C_{1}+B_{1} \tau$.

(ii) (Lipschitz bound in time for $\varphi_{\varepsilon}$ ) Using (44), the first part of (으) and (37) we have

$$
-m_{1}(x) \leq \frac{\partial}{\partial \tau} \varphi_{\varepsilon}=-m_{1}(x)+r(x) \int K(x, y) R_{\varepsilon}(y, \tau) d y \leq \frac{r_{M} R_{1}}{\underline{m}_{2}} .
$$

Since $m_{1}(x)$ is locally bounded, we obtain that $\frac{\partial}{\partial \tau} \varphi_{\varepsilon}$ is locally uniformly bounded. 
(iii) (Convergence of $\varphi_{\varepsilon}$ ) Using (63) and (58) we deduce that $\varphi_{\varepsilon}$ are locally uniformly bounded and its derivatives also. Thanks to the Arzela-Ascoli Theorem, we can extract a subsequence that converges locally uniformly to a continuous function $\varphi$.

Moreover $\varphi$ can not take positive values. Otherwise $\rho_{\varepsilon}=\int n_{\varepsilon} d x$ blows up in the limit as $\varepsilon$ vanishes and this is in contradiction with Theorem 3.1 that states $\rho_{\varepsilon}$ is uniformly bounded.

(iv) (Lipschitz bound in space for $R_{\varepsilon}$ ) We differentiate the second line of (56) with respect to $x$ and we obtain

$$
\nabla R_{\varepsilon}(y, \tau)=\frac{\nabla R_{i n}(y)}{m_{2}(y)+\int r(x) K(x, y) n_{\varepsilon}(x, \tau) d y}-\frac{R_{i n}(y)\left(\nabla m_{2}(y)+\int r(x) K_{y}(x, y) n_{\varepsilon}(x, \tau) d x\right)}{\left(m_{2}(y)+\int r(x) K(x, y) n_{\varepsilon}(x, \tau) d x\right)^{2}} .
$$

Using this equality, (44), (6), (81), the second part of (11) and (16) we have

$$
\begin{aligned}
\left|\nabla R_{\varepsilon}(y, \tau)\right| & \leq \frac{\left|\nabla R_{i n}(y)\right|}{\underline{m}_{2}}+\frac{\left|R_{i n}\right|\left(\left|\nabla m_{2}(y)\right|+r_{M} \sup _{x, y}\left|K_{y}\right| \rho_{\varepsilon}(\tau)\right)}{\underline{m}_{2}^{2}} \\
& \leq \frac{R_{2}}{\underline{m}_{2}}+\frac{R_{1}}{\underline{m}_{2}^{2}}\left(M_{2}+r_{M} K_{3} \rho_{M}\right) .
\end{aligned}
$$

Therefore $R_{\varepsilon}$ is uniformly Lipschitz continuous in space.

It is difficult to obtain time regularity and even in the case of two nutrients, the proof is extremely technical, see [3].

(v) (Convergence of $R_{\varepsilon}$ and identification of the limit) The first parts of (8) and (4) show that $R_{\varepsilon}$ is uniformly bounded. Using this property together with Lipschitz bound in space we obtain that, after extraction of a subsequence, the $R_{\varepsilon}$ 's converge weakly in time and strongly in space.

Now using the strong convergence of $\varphi_{\varepsilon}$ and the weak convergence of $R_{\varepsilon}$ together with (37) we obtain (39).

\section{Proof of Theorem 6.3}

We follow the arguments in [14].

Using (37) and (48) we obtain that $D^{3} \varphi_{\varepsilon}(\tau, x)$ is bounded. Therefore, after extraction of subsequence, the $D^{2} \varphi_{\varepsilon}(\tau, x)$ 's converge locally uniformly to $D^{2} \varphi(\tau, x)$. Using (42) with $\mu_{1} \geq 0$, we obtain that $\varphi_{\varepsilon}$ is concave and thus it has a unique maximum. Now we denote by $\bar{x}_{\varepsilon}(\tau)$ the maximum point of $\varphi_{\varepsilon}$. We have $\nabla \varphi_{\varepsilon}\left(\bar{x}_{\varepsilon}(\tau), \tau\right)=0$ at a maximum point and thus (using the chain rule)

$$
\frac{d}{d \tau} \nabla \varphi_{\varepsilon}\left(\bar{x}_{\varepsilon}(\tau), \tau\right)=0=\frac{\partial}{\partial \tau} \nabla \varphi_{\varepsilon}\left(\bar{x}_{\varepsilon}(\tau), \tau\right)+D^{2} \varphi_{\varepsilon}\left(\bar{x}_{\varepsilon}(\tau), \tau\right) \dot{\bar{x}}_{\varepsilon}(\tau)
$$

From (42) we conclude that $D^{2} \varphi_{\varepsilon}(\bar{x}(\tau), \tau)$ is invertible. Therefore, combining the above equality and (37) we obtain

$$
\begin{aligned}
\frac{d}{d \tau} \bar{x}_{\varepsilon}(\tau) & =\left(-D^{2} \varphi_{\varepsilon}\left(\bar{x}_{\varepsilon}(\tau), \tau\right)\right)^{-1}\left(-\nabla m_{1}\left(\bar{x}_{\varepsilon}(\tau)\right)+r\left(\bar{x}_{\varepsilon}(\tau)\right) \int \nabla_{x} K\left(\bar{x}_{\varepsilon}(\tau), y\right) R_{\varepsilon}(y, \tau) d y\right. \\
& \left.+\nabla r(\bar{x}(\tau)) \int K\left(\bar{x}_{\varepsilon}(\tau), y\right) R_{\varepsilon}(y, \tau) d y\right) .
\end{aligned}
$$

It remains to pass to the limit as $\varepsilon$ vanishes. Firstly, from the strong convexity of $m_{1}$ (from assumption (41) with $\mu_{1}>0$ ) and (37) we conclude that $\bar{x}_{\varepsilon}(\tau)$ is uniformly bounded. Then, from the local bounds 
on $R$, we deduce that $\frac{d}{d \tau} \bar{x}_{\varepsilon}(\tau)$ is also uniformly bounded. It follows again from Arzela-Ascoli Theorem that we may extract a subsequence of $\bar{x}_{\varepsilon}(\cdot)$ that converges uniformly to a Lipschitz continuous function $\bar{x}(\cdot)$. Moreover using that the $D^{2} \varphi_{\varepsilon}$ 's converge locally uniformly to $D^{2} \varphi$, and the $R_{\varepsilon}$ 's converge weakly to $R$ we obtain that

$$
\begin{aligned}
\frac{d}{d \tau} \bar{x}(\tau) & =\left(-D^{2} \varphi(\bar{x}(\tau), \tau)\right)^{-1}\left(-\nabla m_{1}(\bar{x}(\tau))+r(\bar{x}(\tau)) \int \nabla_{x} K(\bar{x}(\tau), y) R(y, \tau) d y\right. \\
& \left.+\nabla r(\bar{x}(\tau)) \int K(\bar{x}(\tau), y) R(y, \tau) d y\right) .
\end{aligned}
$$

Now we prove that $\varphi(\bar{x}(\tau), \tau)=0$ for all $\tau \geq 0$ and that $\bar{\rho}(\tau)>\bar{\rho}(0) e^{-K \tau}$ for all $\tau \geq 0$ and a positive constant $K$. We cannot obtain this directly and thus we begin with proving $l(\tau) \geq 0$, with

$$
l(\tau):=-m_{1}(\bar{x}(\tau))+r(\bar{x}(\tau)) \int K(\bar{x}(\tau), y) \frac{R_{\text {in }}(y)}{m_{2}(y)} d y .
$$

We define

$$
\tau_{0}:=\inf _{s \geq 0}\{l(s)=0\} .
$$

Since $l(\tau)$ is continuous, and $l(0)>0$ according to assumption (49), we have $\tau_{0}>0$. Assume now that $\tau_{0}$ is finite. We have $l(\tau) \geq 0$ in $\left[0, \tau_{0}\right]$. Now we write

$$
\frac{d}{d \tau} \varphi(\bar{x}(\tau), \tau)=\frac{\partial}{\partial \tau} \varphi(\bar{x}(\tau), \tau)=-m_{1}(\bar{x}(\tau))+r(\bar{x}(\tau)) \int K(\bar{x}(\tau), y) \frac{R_{\text {in }}(y)}{m_{2}(y)+\bar{\rho}(\tau) r(\bar{x}(\tau)) K(\bar{x}(\tau), y)} d y
$$

The latter is positive if $\bar{\rho}$ is positive, according to (45). Otherwise it is equal to $l(\tau)$ that is also positive in $\left[0, \tau_{0}\right]$. We obtain that

$$
\frac{d}{d \tau} \varphi(\bar{x}(\tau), \tau) \geq 0, \quad \text { for } \tau \in\left[0, \tau_{0}\right]
$$

Starting with $\varphi(\bar{x}(0), 0)=0$, we deduce that

$$
\varphi(\bar{x}(\tau), \tau)=0, \quad \text { for } \tau \in\left[0, \tau_{0}\right] .
$$

This also shows that the constraint (51) is satisfied and (45) is hold as an equality in $\left(0, \tau_{0}\right)$. Consequently we obtain that $\bar{\rho}$ is in fact a strong limit of $\rho_{\varepsilon}$ in $\left(0, \tau_{0}\right)$.

To gain time regularity, we use (51) and because $\bar{x}(\tau)$ is Lipschitz continuous, we conclude that $\bar{\rho}(\tau)$ and consequently $R(y, \tau)$ are also Lipschitz continuous in $\left(0, \tau_{0}\right)$. Therefore we can differentiate (51) a.e. with respect to $\tau$ and obtain, for all $\tau \in\left[0, \tau_{0}\right)$,

$$
\begin{aligned}
0 & =\left(-\nabla m_{1}\left(\bar{x}(\tau)+r(\bar{x}(\tau)) \int \nabla_{x} K(\bar{x}(\tau), y) \frac{R_{i n}(y)}{m_{2}(y)+\bar{\rho}(t) r(\bar{x}(\tau)) K(\bar{x}(\tau), y)} d y\right) \dot{\bar{x}}(\tau)\right. \\
& \left.+\nabla r(\bar{x}(\tau)) \int K(\bar{x}(\tau), y) \frac{R_{i n}(y)}{m_{2}(y)+\bar{\rho}(t) r(\bar{x}(t)) K(\bar{x}(t), y)} d y\right) \dot{\bar{x}}(\tau) \\
& -\bar{\rho}(\tau) r(\bar{x}(\tau))\left(\int K(\bar{x}(\tau), y) \frac{R_{i n}(y)\left(r(\bar{x}(\tau)) \nabla_{x} K(\bar{x}(\tau), y)+\nabla r(\bar{x}(\tau)) K(\bar{x}(\tau), y)\right)}{\left(m_{2}(y)+\bar{\rho}(\tau) r(\bar{x}(\tau)) K(\bar{x}(\tau), y)\right)^{2}} d y\right) \dot{\bar{x}}(\tau) \\
& -\left(r(\bar{x}(\tau))^{2} \int K(\bar{x}(\tau), y)^{2} \frac{R_{\text {in }}(y)}{\left(m_{2}(y)+\bar{\rho}(\tau) r(\bar{x}(\tau)) K(\bar{x}(\tau), y)\right)^{2}} d y\right) \dot{\bar{\rho}}(\tau) .
\end{aligned}
$$


We deduce that, for a.e. $\tau \in\left(0, \tau_{0}\right)$,

$$
\begin{aligned}
\dot{\bar{x}}(\tau)\left(-D^{2} \varphi(\bar{x}(\tau), \tau) \dot{\bar{x}}(\tau)\right. & =-\dot{\bar{\rho}}(\tau) r(\bar{x}(\tau))^{2} \int K(\bar{x}(\tau), y)^{2} \frac{R_{i n}(y)}{\left(m_{2}(y)+\bar{\rho}(\tau) r(\bar{x}(\tau)) K(\bar{x}(\tau), y)\right)^{2}} d y \\
& -\bar{\rho}(\tau) r(\bar{x}(\tau))\left(\int K(\bar{x}(\tau), y) \frac{R_{\text {in }}(y)\left(r(\bar{x}(\tau)) \nabla_{x} K(\bar{x}(\tau), y)+\nabla r(\bar{x}(\tau)) K(\bar{x}(\tau), y)\right)}{\left(m_{2}(y)+\bar{\rho}(\tau) r(\bar{x}(\tau)) K(\bar{x}(\tau), y)\right)^{2}} d y\right) \dot{\bar{x}}(\tau)
\end{aligned}
$$

It follows that for some constant $K$

$$
\bar{\rho}(0) e^{-K \tau} \leq \bar{\rho}(\tau)
$$

We deduce that $\bar{\rho}(s) \geq \rho_{1}>0$, for $s \in\left(0, \tau_{0}\right)$. Using the constraint and the latter we obtain that, for all $s \in\left[0, \tau_{0}\right)$,

$$
\begin{aligned}
l(s) & \geq l(s)-\left(-m_{1}(\bar{x}(s))+r(\bar{x}(s)) \int K(\bar{x}(s), y) R(s, y) d y\right) \\
& =\bar{\rho}(s) r(\bar{x}(s))^{2} \int K(\bar{x}(s), y)^{2} \frac{R_{\text {in }}(y)}{m_{2}(y)\left(m_{2}(y)+\bar{\rho}(s) r(\bar{x}(s) K(\bar{x}(s), y))\right.} d y \\
& \geq \rho_{1} \int K(\bar{x}(s), y)^{2} \frac{R_{i n}(y)}{C} d y .
\end{aligned}
$$

The latter is bounded from below by a positive constant because $\bar{x}(s)$ is bounded. Using the continuity of $l$ we deduce that $l\left(\tau_{0}\right)>0$. This is impossible and thus contradicts that $\tau_{0}$ is finite.

\section{E Proof of Theorem 7.1}

Firstly we recall the Lipschitz bound (62) proved in Appendix B.

Next, our proof of Theorem 7.1 uses again the equation (61) that we write

$$
\frac{\partial}{\partial t} \varphi(x, t)=-m_{1}(x)+\int \frac{r(x) K(x, y) R_{i n}(y)}{m_{2}(y)+\int r(z) K(z, y) e^{\varphi(z, t)} d z} d y
$$

And we introduce two sets for evaluating the integral in $y$, where $B$ is a constant given later on,

$$
E_{<}=\left\{y ; K(x, y) \leq \frac{B}{2+t}\right\}, \quad E_{>}=\left\{y ; K(x, y) \geq \frac{B}{2+t}\right\}
$$

On $E_{<}$we have

$$
\frac{r(x) K(x, y) R_{i n}(y)}{m_{2}(y)+\int K(z, y) e^{\varphi(z, t)} d z} \leq \frac{r_{M} B}{\underline{m}_{2}(2+t)} R_{\text {in }}(y)
$$


On $E_{>}$we have for some constant $B_{2}, B_{3}$,

$$
\begin{aligned}
\frac{r(x) K(x, y) R_{i n}(y)}{m_{2}(y)+\int r(z) K(z, y) e^{\varphi(z, t)} d z} & \leq e^{-\varphi(x, t)} \frac{r_{M} K(x, y) R_{i n}(y)}{r_{0} \int K(z, y) e^{\varphi(z, t)-\varphi(x, t)} d z} \\
& \leq e^{-\varphi(x, t)} \frac{r_{M} K(x, y) R_{i n}(y)}{r_{0} \int K(z, y) e^{-|x-z|\left(C_{1}+B_{1} t\right)} d z} \\
& \leq e^{-\varphi(x, t)} \frac{r_{M} K(x, y) R_{i n}(y)}{r_{0} \int\left[K(x, y)-K_{2}|x-z|\right] e^{-|x-z|\left(C_{1}+B_{1} t\right)} d z} \\
& \leq e^{-\varphi(x, t)} \frac{K(x, y) R_{i n}(y)}{2 B_{2} \frac{K(x, y)}{2+t}-\frac{B_{3}}{(2+t)^{2}}} \\
& \leq e^{-\varphi(x, t)} \frac{K(x, y) R_{i n}(y)}{B_{2} \frac{K(x, y)}{2+t}}
\end{aligned}
$$

and this leads our choice of $B=B_{3} / B_{2}$.

Altogether, these two controls give

$$
\frac{\partial}{\partial t} \varphi(x, t) \leq M_{i n}\left[\frac{B}{\underline{m}_{2}(2+t)}+\frac{2+t}{B_{2}} e^{-\varphi(x, t)}\right] .
$$

A supersolution to this differential equation is $\psi(t)=A \ln (2+t)$ because for $A$ large enough

$$
\frac{\partial}{\partial t} \psi(t)=\frac{A}{2+t} \geq M_{i n}\left[\frac{B}{\underline{m}_{2}(2+t)}+\frac{1}{B_{2}(2+t)^{(A-1)}}\right], \quad \psi(0) \geq \varphi^{0}(x) .
$$

Therefore for for $A$ large enough, we have $\varphi(x, t) \leq A \ln (t+2)$. Written in terms on $n=e^{\varphi}$ we obtain the conclusion of Theorem 7.1 .

\section{References}

[1] G. Barles and B. Perthame. Concentrations and constrained Hamilton-Jacobi equations arising in adaptive dynamics. Contemp. Math., 439:57-68, 2007.

[2] J. A. Carrillo, S. Cuadrado, and B. Perthame. Adaptive dynamics via Hamilton-Jacobi approach and entropy methods for a juvenile-adult model. Math. Biosci., 205(1):137-161, 2007.

[3] N. Champagnat and P.-E. Jabin. The evolutionary limit for models of populations interacting competitively with many resources. Preprint, 2010.

[4] L. Desvillettes, P.-E. Jabin, S. Mischler, and G. Raoul. On mutation-selection dynamics. Commun. Math. Sci., 6(3):729-747, 2008.

[5] U. Dieckmann and R. Law. The dynamical theory of coevolution: A derivation from stochastic ecological processes. J. Math. Biol., 34:579-612, 1996.

[6] O. Diekmann. A beginner's guide to adaptive dynamics. In Mathematical modelling of population dynamics, volume 63 of Banach Center Publ., pages 47-86. Polish Acad. Sci., Warsaw, 2004. 
[7] O. Diekmann, P.-E. Jabin, S. Mischler, and B. Perthame. The dynamics of adaptation: an illuminating example and a Hamilton-Jacobi approach. Th. Pop. Biol., 67(4):257-271, 2005.

[8] S. Génieys, V. Volpert, and P. Auger. Pattern and waves for a model in population dynamics with nonlocal consumption of resources. Math. Model. Nat. Phenom., 1(1):63-80, 2006.

[9] S. A. H. Geritz, E. Kisdi, G. Mészena, and J. A. J. Metz. Evolutionarily singular strategies and the adaptive growth and branching of the evolutionary tree. Evol. Ecol, 12:35-57, 1998.

[10] S. A. H. Geritz, J. A. J. Metz, E. Kisdi, and G. Meszéna. Dynamics of adaptation and evolutionary branching. Phys. Rev. Lett., 78(10):2024-2027, Mar 1997.

[11] C. H. Ito and U. Dieckmann. A new mechanism for recurrent adaptive radiations. Amer. Nat., 170(4):E96-E111, 2007.

[12] P.-E. Jabin and G. Raoul. Selection dynamics with competition. J. Math. Biol., To appear.

[13] S. A. Levin. Community equilibria and stability, and an extension of the competitive exclusion principle. The American Naturalist, 104:413-423, 1970.

[14] A. Lorz, S. Mirrahimi, and B. Perthame. Dirac mass dynamics in multidimensional nonlocal parabolic equations. Comm. Partial Differential Equations, To appear.

[15] R. MacArthur. Species packing and competetive equilibrium for many species. Th. Pop. Biol., 1:1-11, 1970.

[16] J. A. J. Metz, Geritz S. A. H., G. Meszéna, Jacobs F. J. A., and J.S. Van Heerwaarden. Adaptive dynamics, a geometrical study of the consequences of nearly faithful reproduction. In S. J. Van Strien and S. M. Verduyn Lunel, editors, Stochastic and spatial structures of dynamical systems., pages 183-231. North Holland, Elsevier, 1996.

[17] B. Perthame. Transport equations in biology. Frontiers in Mathematics. Birkhäuser Verlag, Basel, 2007.

[18] B. Perthame and G. Barles. Dirac concentrations in Lotka-Volterra parabolic PDEs. Indiana Univ. Math. J., 57(7):3275-3301, 2008.

[19] G. Raoul. Etude qualitative et numérique d'équations aux dérivées partielles issues des sciences de la nature. PhD thesis, ENS Cachan, 2009.

[20] G. Raoul. Local stability of evolutionary attractors for continuous structured populations. accepted in Monatsh. Math., 2010.

[21] A. Sasaki. Clumped distribution by neighborhood competition. Journal of Theoretical Biology, 186(4):415-430, 1997.

[22] A. Sasaki and S. Ellner. The evolutionarily stable phenotype distribution in a random environment. Evolution, 49(2):337-350, 1995.

[23] T. W. Schoener. Resource partitioning in ecological communities. Science, 13:27-39, 1974.

[24] R. W. Sterner and J. J. Elser. Ecological Stoichiometry: The Biology of Elements from Molecules to the Biosphere. Princeton University Press, 2002. 